\title{
Efficient Two-Round OT Extension and Silent Non-Interactive Secure Computation
}

\author{
Elette Boyle \\ IDC Herzliya \\ Israel \\ eboyle@alum.mit.edu \\ Yuval Ishai \\ Technion \\ Israel \\ yuvali@cs.technion.ac.il
}

\author{
Geoffroy Couteau \\ Karlsruhe Institute of Technology \\ Germany \\ geoffroy.couteau@kit.edu \\ Lisa Kohl \\ Karlsruhe Institute of Technology \\ Germany \\ lisa.kohl@kit.edu \\ Peter Scholl \\ Aarhus University \\ Denmark \\ peter.scholl@cs.au.dk
}

\author{
Niv Gilboa \\ Ben Gurion University \\ Israel \\ gilboan@bgu.ac.il \\ Peter Rindal \\ Visa Research \\ rindalp@oregonstate.edu
}

\begin{abstract}
We consider the problem of securely generating useful instances of two-party correlations, such as many independent copies of a random oblivious transfer (OT) correlation, using a small amount of communication. This problem is motivated by the goal of secure computation with silent preprocessing, where a low-communication input-independent setup, followed by local ("silent") computation, enables a lightweight "non-cryptographic" online phase once the inputs are known.

Recent works of Boyle et al. (CCS 2018, Crypto 2019) achieve this goal with good concrete efficiency for useful kinds of two-party correlations, including OT correlations, under different variants of the Learning Parity with Noise (LPN) assumption, and using a small number of "base" oblivious transfers. The protocols of Boyle et al. have several limitations. First, they require a large number of communication rounds. Second, they are only secure against semi-honest parties. Finally, their concrete efficiency estimates are not backed by an actual implementation. In this work we address these limitations, making three main contributions:
\end{abstract}

- Eliminating interaction. Under the same assumption, we obtain the first concretely efficient 2-round protocols for generating useful correlations, including OT correlations, in the semi-honest security model. This implies the first efficient 2-round OT extension protocol of any kind and, more generally, protocols for non-interactive secure computation (NISC) that are concretely efficient and have the silent preprocessing feature.

Permission to make digital or hard copies of all or part of this work for personal or classroom use is granted without fee provided that copies are not made or distributed for profit or commercial advantage and that copies bear this notice and the full citation on the first page. Copyrights for components of this work owned by others than the author(s) must be honored. Abstracting with credit is permitted. To copy otherwise, or republish, to post on servers or to redistribute to lists, requires prior specific permission and/or a fee. Request permissions from permissions@acm.org.

CCS '19, November 11-15, 2019, London, United Kingdom

(C) 2019 Copyright held by the owner/author(s). Publication rights licensed to ACM. ACM ISBN 978-1-4503-6747-9/19/11 . \$ \$15.00

https://doi.org/10.1145/3319535.3354255
- Malicious security. We provide security against malicious parties without additional interaction and with only a modest overhead; prior to our work, no similar protocols were known with any number of rounds.

- Implementation. Finally, we implemented, optimized, and benchmarked our 2-round OT extension protocol, demonstrating that it offers a more attractive alternative to the OT extension protocol of Ishai et al. (Crypto 2003) in many realistic settings.

\section{CCS CONCEPTS}

- Security and privacy $\rightarrow$ Cryptography.

\section{KEYWORDS}

secure computation; oblivious transfer extension; pseudorandom correlation generators; active security

\section{ACM Reference Format:}

Elette Boyle, Geoffroy Couteau, Niv Gilboa, Yuval Ishai, Lisa Kohl, Peter Rindal, and Peter Scholl. 2019. Efficient Two-Round OT Extension and Silent Non-Interactive Secure Computation. In 2019 ACM SIGSAC Conference on Computer and Communications Security (CCS '19), November 11-15, 2019, London, United Kingdom. ACM, New York, NY, USA, 18 pages. https://doi. org $/ 10.1145 / 3319535.3354255$

\section{INTRODUCTION}

There is a large body of work on optimizing the concrete efficiency of secure computation protocols via input-independent preprocessing. By securely generating many instances of simple correlations, one can dramatically reduce the online communication and computation costs of most existing protocols.

To give just one example, multiple independent instances of a random oblivious transfer ${ }^{1}$ (OT) correlation can be used for secure two-party computation of Boolean circuits in the semi-honest model, with communication cost of only two bits per party per

\footnotetext{
${ }^{1}$ In (a single instance of) a random OT correlation, one party obtains a pair of random bits (more generally, strings) $\left(s_{0}, s_{1}\right)$ and the other obtains the pair $\left(r, s_{r}\right)$ for a random bit $r$.
} 
(nonlinear) gate, and with computation cost that is comparable to computing the circuit with no security requirements at all [9, 33, 43] Thus, assuming a fast communication network, protocols based on correlated randomness can achieve near-optimal performance.

The main challenge in applying this approach is the high concrete cost of securely generating the correlated randomness. Traditional solutions involve carefully optimized special-purpose secure computation protocols that have a high communication cost for each instance of the desired correlation [11,22]. This holds even for the case of OT correlations, for which relatively fast OT extension techniques are known $[8,37,41]$. Moreover, even if offline communication is cheap, the cost of storing large amounts of correlated randomness for each party with whom a future interaction might take place can be significant.

Motivated by the limitations of traditional approaches for generating and storing correlated randomness, the notion of a pseudorandom correlation generator (PCG) was recently proposed and studied by Boyle et al. $[15,16]$. The goal of a PCG is to compress long sources of correlated randomness without violating security. More concretely, a (two-party) PCG replaces a target two-party correlation, say many independent OT correlation instances, by a pair of short correlated keys, which can be "silently" expanded without any interaction. The process of generating the correlated keys and locally expanding them should emulate an ideal process for generating the target correlation not only from the point of view of outsiders, but also from the point of view of insiders who can observe one of the two keys. Among other results, the aforementioned works of Boyle et al. $[15,16]$ obtain concretely efficient constructions of PCGs for OT correlations and vector oblivious linear evaluation (VOLE) correlations $[4,39,48]$ based on variants of the Learning Parity with Noise assumption [12]. These PCG constructions are motivated by the goal of secure computation with silent preprocessing, where a low-communication input-independent setup, followed by local ("silent") computation, enables a lightweight "non-cryptographic" online phase once the inputs are known.

However, towards realizing this goal, one major challenge remains: how can the pair of keys be securely generated? While the keys are short, their sampling algorithm is quite complex and involves multiple invocations of cryptographic primitives. Thus, even applying the fastest general-purpose protocols (e.g., [40]) for generating these keys incurs a very significant overhead.

An alternative approach for distributing the PCG key generation, suggested in $[15,16]$, relies on a recent special-purpose protocol of Doerner and shelat [24] for secure key generation of a distributed point function (DPF) $[17,30]$. This protocol only makes a black-box use of symmetric cryptography and a small number of oblivious transfers, and hence it is also concretely efficient. Using this protocol for distributing the key generation of a PCG for OT correlations, Boyle et al. [16] obtained a silent OT extension protocol that generates (without any trusted setup) a large number of pseudo-random OTs from a small number of base OTs, using a low-communication setup followed by silent key expansion [16].

While the silent OT extension protocol from [16] and other protocols obtained using this approach have good concrete efficiency, they also have several limitations. First, they require a large number of communication rounds that grows (at least) logarithmically with the output length. Second, they are only secure against semihonest parties. Both of the above limitations are inherited from the DPF key generation protocol of [24]. Finally, their concrete efficiency estimates are not backed by an actual implementation, and ignore possible cache-misses and other system- and networkrelated sources of slowdown.

\subsection{Our Contribution}

In this work, we address the above limitations by making the following contributions.

Two-Round Silent OT Extension. We present the first concretely efficient two-round OT extension protocol, based on a variant of the LPN assumption. The protocol has a silent preprocessing feature, allowing the parties to push the bulk of the computational work to an offline phase. It can be used in two modes: either a random-input mode, where the communication complexity is sublinear in the outputs length, or a chosen-input mode, where the communication is roughly equal to the total input length. This applies even to the more challenging case of 1-bit OT, for which standard OT extension techniques that make a black-box use of symmetric cryptography $[8,37,41,44]$ have a high communication overhead compared to the input length. A key idea that underlies this improvement is replacing the DPF primitive in the PCG for OT from [16] by the simpler puncturable pseudorandom function (PPRF) primitive $[14,18,42]$. We design a parallel version of the distributed key generation protocol from [24] that applies to a PPRF instead of a DPF.

Our OT extension protocol bypasses a recent impossibility result of Garg et al. [28] on 2-round OT extension due to the use of the LPN assumption. While our construction (inevitably) does not fall into the standard black-box framework considered [28], it still has a black-box flavor in that it only invokes a syndrome computation of any error-correcting code for which the LPN assumption holds. We remark that aside from its concrete efficiency, our 2-round OT extension protocol can be based on a conservative variant of (binary) LPN in a noise regime that is not known to imply public-key encryption, let alone oblivious transfer [3].

The technique we use for generating OT correlations in two rounds can also be applied to VOLE correlations, as well as general protocols for non-interactive secure computation (NISC) with silent preprocessing.

Malicious Security. We present simple, practical techniques for secure distributed setup of PPRF keys with a weak form of malicious security. This suffices to upgrade our semi-honest OT and VOLE protocols to malicious security, at a very low cost. Our main protocols in this setting have 4 rounds of interaction, but this can be reduced to 2 rounds using the Fiat-Shamir transform. We can also use this to obtain maliciously secure silent NISC or two-round OT extension on chosen inputs. These protocols are based on slightly stronger variants of LPN, where the adversary is allowed a single query to a one-bit leakage function on the error vector.

Implementation. We demonstrate the efficiency of our constructions with an implementation of our random OT extension protocol. The most costly part of the implementation is a large matrix-vector multiplication, which comes from applying the LPN assumption. 
We optimize this using quasi-cyclic codes, similarly to several recent, candidate post-quantum secure cryptosystems, and present different tradeoffs with parameter choices. Our protocols have a very low communication overhead and perform significantly faster than previous, state-of-the-art protocols $[8,37,41]$ in environments with restricted bandwidth. For instance, in a 100Mbps WAN setting, we are around $5 \mathrm{x}$ faster, and this improves to $47 \mathrm{x}$ in a $10 \mathrm{MBps}$ WAN. This is because, while our computational costs are around an order of magnitude higher, we need around 1000-2000 times less communication than the other protocols.

Applications. As well as the new application to NISC with silent preprocessing, our protocols can be applied to a range of traditional secure computation tasks. Below we mention just a few areas where we expect silent OT extension to have an impact.

- Semi-honest MPC. In the semi-honest "GMW protocol” [33], the correlated randomness needed to evaluate a Boolean circuit can be obtained from two random OTs per AND gate. Plugging in our random OT extension, we obtain a practical 2-PC protocol where each party communicates just 2 bits per AND gate on average. This is around $30 \mathrm{x}$ less communication than the state-of-the-art [23].

- Malicious secure MPC. Protocols based on authenticated garbling $[55,56]$ are currently the state-of-the-art in maliciously secure MPC for binary circuits, particularly in a highlatency scenario. The main cost in these protocols comes from a preprocessing phase, where the parties use a large number of random, correlated oblivious transfers to produce correlated randomness. Our protocol can produce the same kind of oblivious transfers with almost zero communication, and we estimate this could reduce the overall communication in authenticated garbling protocols by around an order of magnitude.

- Private set intersection (PSI). In circuit-based PSI, a generic 2-PC protocol is used to first compute a secret-sharing of the intersection of two sets, and then perform some useful computation on the result $[36,50,51]$. With the improvements to GMW mentioned above, we can expect to obtain a similar reduction in communication for these families of PSI protocols.

\subsection{Technical Overview}

We now give an overview of our silent constructions in the semihonest and malicious settings. For simplicity, we focus here on the case of 1-out-of-2 oblivious transfer.

We start by recalling the high-level idea of the pseudorandom correlation generators for vector-OLE (VOLE) and OT from $[15,16]$. These constructions distribute a pair of seeds to a sender and a receiver, who can then locally expand the seeds to produce many instances of pseudorandom OT or VOLE. To do so, they use two main ingredients: a variant of the LPN assumption, and a method for the two parties to obtain a compressed form of random secret shares $\vec{v}_{0}, \vec{v}_{1}$, satisfying

$$
\vec{v}_{1}=\vec{v}_{0}+\vec{e} \cdot x \in \mathbb{F}_{2^{\lambda}}^{N}
$$

where $\vec{e} \in\{0,1\}^{N}$ is a random, sparse vector held by one party, and $x \in \mathbb{F}_{2^{\lambda}}$ is a random field element held by the other party.

Given this, the shares can be randomized by taking a public, binary matrix $H$ that compresses from $N$ down to $n<N$ elements, and locally multiplying each share with $H$. This works because $\vec{u}=\vec{e} \cdot H$ is pseudorandom under a suitable variant of LPN. Writing $\vec{v}=\vec{v}_{0} \cdot H$ and $\vec{w}=\vec{v}_{1} \cdot H$, from (1) we then get $\vec{w}=\vec{v}+\vec{u} x$. This can be seen as a set of random correlated OTs, where $u_{i} \in\{0,1\}$ are the receiver's choice bits, and $\left(v_{i}, v_{i}+x\right)$ are the sender's strings, of which the receiver learns $w_{i}$. These can be locally converted into random string-OTs with a standard hashing technique [37].

To obtain a compressed form of the shares in (1), the constructions of $[15,16]$ used a distributed point function (DPF) [17, 30]. Our first observation is that the distributed point function is an overkill for this application, ${ }^{2}$ and can be replaced with the simpler puncturable pseudorandom function (PPRF) primitive. A PPRF is a PRF $F$ such that given an input $x$, and a PRF key k, one can generate a punctured key $\mathrm{k}\{x\}$ which allows evaluating $F$ at every point except for $x$, and does not conceal any information about the value $F(\mathrm{k}, x)$. A PPRF can be built from any length-doubling pseudorandom generator, using a binary tree-based construction [14, 18, 42].

In the setup procedure, we will give the sender a random key $\mathrm{k}$ and $x$, and give to the receiver a random point $\alpha \in\{1, \ldots, N\}$, a punctured key $\mathrm{k}\{\alpha\}$, and the value $z=F(\mathrm{k}, \alpha)+x$. Given these seeds, the sender and receiver can now define the expanded outputs, for $i=1, \ldots, n$ :

$$
\vec{v}_{0}[i]=F(\mathrm{k}, i), \quad \vec{v}_{1}[i]= \begin{cases}F(\mathrm{k}, i) & i \neq \alpha \\ z & \text { otherwise }\end{cases}
$$

These immediately satisfy (1), with $\vec{e}$ as the $\alpha$-th unit vector. To obtain sharings of sparse $\vec{e}$ with, say, $t$ non-zero coordinates, as needed to use LPN, we repeat this $t$ times and XOR together all $t$ sets of outputs.

Conceptually, this construction is simpler than using a DPF, and moreover, as we now show, it brings several efficiency advantages.

Two-Round Setup of Puncturable PRF Keys. We present a simple, two-round protocol for distributed the above setup with semihonest security, inspired by the DPF setup protocol of Doerner and shelat [24]. The core of our protocol is the following procedure. For each of $t$ secret LPN noise coordinates $\alpha_{j} \in[N]$ known to the receiver, the sender generates a fresh PRF key $\mathrm{k}_{j}$, and wishes to obliviously communicate a punctured key $\mathrm{k}_{j}\left\{\alpha_{j}\right\}$ and hardcoded punctured output $z_{j}=\operatorname{PRF}\left(\mathrm{k}_{j}, \alpha\right)+x$ to the receiver. Combined, this yields a secret sharing of the vector $x \cdot \vec{e}$, as required. To do so, for each $\mathrm{k}\{\alpha\}$, the parties made use of $\ell=\log N$ parallel OT executions: the sender's $\ell$ message pairs correspond to appropriate sums of partial evaluations from a consistent GGM PRF tree and his secret value $x$, and the receiver's $\ell$ selection bits correspond to the bits of his chosen path $\alpha$.

Compared with previous works based on distributed point functions $[15,16,24]$, the number of rounds of interaction collapses from $O(\log N)$ to just two, given any two-round OT protocol. This is possible since the punctured point $\alpha$ is known to the receiver,

\footnotetext{
${ }^{2}$ In contrast, we do not know how to replace DPF by PPRF in some of the other PCG constructions from [16], including the LPN-based constructions for low-degree correlations and the PRG-based constructions for one-time-truth-table correlations.
} 
whereas when $\alpha$ is secret-shared as in a DPF, the OTs in the setup procedure seem hard to parallelize.

Two-Round OT Extension and Silent NISC. We observe that in the two-round setup, the receiver can already compute part of its output before sending the first round message. In the case of OT, this part corresponds to its random vector of choice bits $\vec{u}$. This means that the receiver can already derandomize its OT outputs in the first round, by sending in parallel with its setup message the value $\vec{u}+\vec{c}$, where $\vec{c}$ is its chosen input vector. Since the sender can compute its random OT outputs after the first round, this leads to a tworound OT extension protocol that additionally enjoys the "silent preprocessing" feature of pushing the bulk of the computation to an offline phase, before the inputs are known. This can be generalized from OT to VOLE and other useful instances of non-interactive secure computation (NISC) [38], simultaneously inheriting the silent preprocessing feature from the PCG and the interaction feature from an underlying NISC protocol. See Section 3 for a more detailed discussion of our new notion of NISC with silent preprocessing.

Maliciously Secure Setup. In the above semi-honest setup procedure, a malicious receiver has no cheating space; altered selection bits merely correspond to a different choice of noise coordinate $\alpha^{\prime} \in[N]$. However, a malicious sender may generate message pairs inconsistent with any correct PRF evaluation tree, or use inconsistent inputs $x$ across the $t$ executions (in which case the outputs are not valid shares of $x \cdot \vec{u}$ for any single $x$ ). For example, by injecting errors into one of the two messages within an OT message pair, the sender can effectively "guess" and learn a bit of $\alpha$, and will go unnoticed if his guess is correct.

We demonstrate that with small overhead, we can restrict a malicious sender to only such selective-failure attacks. This is formalized via an ideal functionality where the adversarial sender can send a guess range $I \subseteq[N]$ for $\alpha$, a "getting caught" predicate is tested as a function of the receiver's true input, and the functionality either aborts or delivers the output accordingly. We then show that paired with an interactive leakage notion for LPN, this suffices to give us PCG setup protocols for VOLE and OT with malicious security.

Our basic maliciously secure protocols have 4 rounds, but this can be compressed to two rounds with the Fiat-Shamir transform, in the random oracle model. Just as in the semi-honest protocols, we can convert the setup protocols into NISC protocols, this time under a slightly stronger variant of LPN with one bit of adaptive leakage on the error vector, obtaining two-round OT extension with malicious security.

\section{PRELIMINARIES}

\subsection{Puncturable Pseudorandom Function}

Pseudorandom functions (PRF) are keyed functions which are indistinguishable from truly random functions, have been introduced in [32]. A puncturable pseudorandom function (PPRF) is a PRF $F$ such that given an input $x$, and a PRF key $k$, one can generate a punctured key, denoted $k\{x\}$, which allows evaluating $F$ at every point except for $x$, and does not conceal any information about the value of $F$ at $x$. PPRFs have been introduced in [14, 18, 42].

Definition 2.1 ( $t$-Puncturable Pseudorandom Function). A puncturable pseudorandom function (PPRF) with key space $\mathcal{K}$, domain

\section{Experiment Exp-s-pPRF}

Setup Phase. The adversary $\mathcal{A}$ sends a size- $t$ subset $S^{*} \in \mathcal{X}$ to the challenger. When it receives $S^{*}$, the challenger picks $K \stackrel{\$}{\leftarrow} F$.KeyGen $\left(1^{\lambda}\right)$ and a random bit $b \stackrel{\$}{\leftarrow}\{0,1\}$.

Challenge Phase. The challenger sends $K\left\{S^{*}\right\}$ $F$.Puncture $\left(K, S^{*}\right)$ to $\mathcal{A}$. If $b=0$, the challenger additionally sends $(F(K, x))_{x \in S^{*}}$ to $\mathcal{A}$; otherwise, if $b=1$, the challenger picks $t$ random values $\left(y_{x} \stackrel{\$}{\leftarrow} \mathcal{Y}\right.$ for every $x \in S^{*}$ ) and sends them to $\mathcal{A}$.

Figure 1: Selective security game for puncturable pseudorandom functions. At the end of the experiment, $\mathcal{A}$ sends a guess $b^{\prime}$ and wins if $b^{\prime}=b$.

$\mathcal{X}$, and range $\mathcal{Y}$, is a pseudorandom function $F$ with an additional punctured key space $\mathcal{K}_{p}$ and three probabilistic polynomial-time algorithms ( $F$.KeyGen, $F$.Puncture, $F$.Eval) such that

- F.KeyGen $\left(1^{\lambda}\right)$ outputs a random key $K \in \mathcal{K}$,

- F.Puncture $(K, S)$, on input a key $K \in \mathcal{K}$, and a subset $S \subset \mathcal{X}$ of size $t$, outputs a punctured key $K\{S\} \in \mathcal{K}_{p}$,

- $F$.Eval $(K\{S\}, x)$, on input a key $K\{S\}$ punctured at all points in $S$, and a point $x$, outputs $F(K, x)$ if $x \notin S$, and $\perp$ otherwise,

such that no probabilistic polynomial-time adversary wins the experiment Exp-s-pPRF represented on Figure 1 with non-negligible advantage over the random guess.

By $F$.FullEval $(K)$ we denote the algorithm that on input a key $K \in \mathcal{K}$ evaluates $F$ on all inputs $\mathcal{X}$ and returns the vector of outputs.

A PPRF can be constructed from any length-doubling pseudorandom generator, using the GGM tree-based construction [14, $18,32,42]$. The construction proceeds as follows: On input a key $K$ and a point $x$, set $K^{(0)} \leftarrow K$ and perform the following iterative evaluation procedure: for $i=1$ to $\ell \leftarrow \log |x|$, compute $\left(K_{0}^{(i)}, K_{1}^{(i)}\right) \leftarrow G\left(K^{(i-1)}\right)$, and set $K^{(i)} \leftarrow K_{x_{i}}^{(i)}$. Output $K^{(\ell)}$. This procedure creates a complete binary tree with edges labeled by keys; the output of the PRF on an input $x$ is the key labeling the leaf at the end of the path defined by $x$ from the root of the tree.

- F.KeyGen $\left(1^{\lambda}\right)$ : output a random seed for $G$.

- F.Puncture $(K, z)$ : on input a key $K \in\{0,1\}^{k}$ and a point $x$, apply the above procedure and return $K\{x\}=\left(K_{1-x_{1}}^{(1)}, \ldots\right.$, $\left.K_{1-x_{\ell}}^{(\ell)}\right)$.

- F.Eval $\left(K\{x\}, x^{\prime}\right)$, on input a punctured key $K\{x\}$ and a point $x$, if $x=x^{\prime}$, output $\perp$. Otherwise, parse $K\{x\}$ as $\left(K_{1-x_{1}}^{(1)}, \ldots\right.$, $\left.K_{1-x_{\ell}}^{(\ell)}\right)$ and start the iterative evaluation procedure from the first $K_{1-x_{i}}^{(i)}$ such that $x_{i}^{\prime}=1-x_{i}$.

To obtain a $t$-puncturable PRF with input domain $[n]$, one can simply run $t$ instances of the above puncturable PRF and set the output of the PRF to be the bitwise xor of the output of each instance. With this construction, the length of a key punctured at $t$ points is $t \lambda \log n$, where $\lambda$ is the seed size of the PRG. 


\subsection{Learning Parity with Noise}

In this work, we rely on variants of the Learning Parity with Noise (LPN) assumption [12] over either $\mathbb{F}_{2}$ or a large finite field $\mathbb{F}$, where the noise is assumed to have a small Hamming weight. Similar assumptions have been used in the context of secure arithmetic computation [4, 25, 29, 39, 48]; unlike most of these works, the flavors of LPN on which we rely do not require the underlying code to have an algebraic structure and are thus not susceptible to algebraic (list-) decoding attacks.

Definition $2.2(L P N)$. Let $\mathcal{D}(\mathcal{R})=\left\{\mathcal{D}_{k, q}(\mathcal{R})\right\}_{k, q \in \mathbb{N}}$ denote a family of distributions over a ring $\mathcal{R}$, such that for any $k, q \in \mathbb{N}$, $\operatorname{Im}\left(\mathcal{D}_{k, q}(\mathcal{R})\right) \subseteq \mathcal{R}^{q}$. Let $\mathrm{C}$ be a probabilistic code generation algorithm such that $\mathrm{C}(k, q, \mathcal{R})$ outputs a matrix $A \in \mathcal{R}^{k \times q}$. For dimension $k=k(\lambda)$, number of samples (or block length) $q=q(\lambda)$, and ring $\mathcal{R}=\mathcal{R}(\lambda)$, the $(\mathcal{D}, \mathrm{C}, \mathcal{R})$ - $\mathrm{LPN}(k, q)$ assumption states that

$$
\begin{aligned}
& \left\{(A, \vec{b}) \mid A \stackrel{\$}{\leftarrow} \mathrm{C}(k, q, \mathcal{R}), \vec{e} \stackrel{\$}{\leftarrow} \mathcal{D}_{k, q}(\mathcal{R}), \vec{s} \stackrel{\$}{\leftarrow} \mathbb{F}^{k}, \vec{b} \leftarrow \vec{s} \cdot A+\vec{e}\right\} \\
& \stackrel{\mathrm{c}}{\approx}\left\{(A, \vec{b}) \mid A \stackrel{\$}{\leftarrow} \mathrm{C}(k, q, \mathcal{R}), \vec{b} \stackrel{\$}{\leftarrow} \mathcal{R}^{q}\right\}
\end{aligned}
$$

Here and in the following, all parameters are functions of the security parameter $\lambda$ and computational indistinguishability is defined with respect to $\lambda$. When $\mathcal{R}=\mathbb{F}_{2}$ and $\mathcal{D}$ is the Bernoulli distribution over $\mathbb{F}_{2}^{q}$, where each coordinate is 1 with probability $r$ and 0 otherwise, this corresponds to the standard binary LPN assumption. Note that the search LPN problem, of finding the vector can be reduced to the decisional LPN assumption as defined above above when the code generator $\mathrm{C}$ outputs a uniform matrix $A[5,12]$. However, this is less relevant for us as we are mainly interested in efficient variants with more structured codes. See [26] for further discussion of search-to-decision reductions in the general case.

Example: LPN with Fixed Weight Noise. For a finite field $\mathbb{F}$, we denote by $\mathcal{H} \mathcal{W}_{r}(\mathbb{F})$ the distribution of uniform, weight $r$ vectors over $\mathbb{F}$; that is, a sample from $\mathcal{H} \mathcal{W}_{r}(\mathbb{F})$ is a uniformly random nonzero field element in $r$ random positions, and zero elsewhere. The $\left(\operatorname{Ber}_{r}(\mathbb{F})^{q}, \mathbf{C}, \mathbb{F}\right)-\operatorname{LPN}(k, q)$ assumption corresponds to the standard (non-binary, fixed-weight) LPN assumption over a field $\mathbb{F}$ with code generator $\mathrm{C}$, dimension $k$, number of samples (or block length) $q$, and noise rate $r$.

When the block length $q$ and noise rate $r$ are such that $k$ random coordinates will be all noiseless with non-negligible probability (e.g., when $r$ is constant and $q=\Omega\left(k^{2}\right)$ ), LPN can be broken via Gaussian elimination (cf. [7]). This attack does not apply to our constructions, which typically have $q=O(k)$.

Definition 2.3 (dual LPN). Let $\mathcal{D}(\mathcal{R})$ and $\mathrm{C}$ be as in Definition 2.2, $n, N \in \mathbb{N}$ with $N>n$, and define $C^{\perp}(N, n, \mathcal{R})=\left\{B \in \mathcal{R}^{N \times n}\right.$ : $A \cdot B=0, A \in \mathrm{C}(N-n, N, \mathcal{R}), \operatorname{rank}(B)=n\}$.

For $n=n(\lambda), N=N(\lambda)$ and $\mathcal{R}=\mathcal{R}(\lambda)$, the $(\mathcal{D}, \mathrm{C}, \mathcal{R})$-dual-LPN $(N, n)$ assumption states that

$$
\begin{aligned}
\{(H, \vec{b}) \mid H & \left.\stackrel{\$}{\leftarrow} \mathrm{C}^{\perp}(N, n, \mathcal{R}), \vec{e} \stackrel{\$}{\leftarrow} \mathcal{D}(\mathcal{R}), \vec{b} \leftarrow \vec{e} \cdot H\right\} \\
& \stackrel{c}{\approx}\left\{(H, \vec{b}) \mid H \stackrel{\$}{\leftarrow} \mathrm{C}^{\perp}(N, n, \mathcal{R}), \vec{b} \stackrel{\$}{\leftarrow} \mathcal{R}^{n}\right\}
\end{aligned}
$$

We will slightly abuse our notations by omitting to explicitely mention the code $\mathrm{C}$ and writing $(\mathcal{D}, H, \mathcal{R})$-dual-LPN $(N, n)$ for above dual-LPN assumption with a matrix $H \in \mathrm{C}^{\perp}(N, n, \mathcal{R})$.
The search version of the dual LPN problem is also known as syndrome decoding. The decision version defined above is equivalent to primal variant of LPN from Definition 2.2 with dimension $N-n$ and number of samples $N$. This follows from the simple fact that $(\vec{s} \cdot A+\vec{e}) \cdot H=\vec{s} \cdot A \cdot H+\vec{e} \cdot H=\vec{e} \cdot H$, when $H$ is the parity-check matrix of $A$.

Attacks on LPN. We recall here the main attacks on LPN, following the analysis of [15]. We refer the reader to [27] for a more comprehensive overview. We assume that $\mathcal{D}$ is a noise distribution with Hamming weight bounded by some integer $t$.

- Gaussian elimination. The most natural attack on LPN recovers $\vec{s}$ from $\vec{b}=\vec{s} \cdot A+\vec{e}$ by guessing $n$ non-noisy coordinates of $\vec{b}$, and inverting the corresponding subsystem to verify whether the guess was correct. This approach recovers $\vec{s}$ in time at least $(1 /(1-r))^{n}$ using at least $O(n / r)$ samples $(r=t / N)$. For low-noise LPN, with noise rate $1 / n^{c}$ for some constant $c \geq 1 / 2$, this translates to a bound on attacks of $O\left(e^{n^{1-c}}\right)$ time using $O\left(n^{1+c}\right)$ samples.

- Information Set Decoding (ISD) [52]. Breaking LPN is equivalent to solving its dual variant, which can be interpreted as the task of decoding a random linear code from its syndrome. The best algorithms for this task are improvements of Prange's ISD algorithm, which attempts to find a size- $t$ subset of the rows of $B$ (the parity-check matrix of the code) that spans $\vec{e} \cdot B$, where $t=r N$ is the number of noisy coordinates. The state of the art variant of Prange's information set decoding attack is the BJMM attack [10], which was analyzed in [54], and in the NIST candidate BIKE [6, Section 5.2], which also take into account the effect of the DOOM attack [53] which applies to the specific case of LPN with quasi-cyclic codes.

- The BKW algorithm [13]. This algorithm is a variant of Gaussian elimination which achieves subexponential complexity even for high-noise LPN (e.g. constant noise rate), but requires a subexponential number of samples: the attack solves LPN over $\mathbb{F}_{2}$ in time $2^{O(n / \log (n / r))}$ using $2^{O(n / \log (n / r))}$ samples.

- Combinations of the above [27]. The authors of [27] conducted an extended study of the security of LPN, and described combinations and refinements of the previous three attacks (called the well-pooled Gauss attack, the hybrid attack, and the well-pooled MMT attack). All these attacks achieve subexponential time complexity, but require as many sample as their time complexity.

- Scaled-down BKW [45]. This algorithm is a variant of the BKW algorithm, tailored to LPN with polynomially-many samples. It solves LPN in time $2^{O(n / \log \log (n / r))}$, using $n^{1+\varepsilon}$ samples (for any constant $\varepsilon>0$ ) and has worse performance in time and number of samples for larger fields.

- Low-Weight Parity Check [57]. Eventually, all the previous attacks recover the secret $\vec{s}$. A more efficient attack (by a polynomial factor) can be used if one simply wants to distinguish $\vec{b}=\vec{s} \cdot A+\vec{e}$ from random: by the singleton bound, the minimal distance of the dual code of $\mathrm{C}$ is at most $n+1$, hence there must be a parity-check equation for $\mathrm{C}$ of 
weight $n+1$. Then, if $\vec{b}$ is random, it passes the check with probability at most $1 /|\mathbb{F}|$, whereas if $\vec{b}$ is a noisy encoding, it passes the check with probability at least $((N-n-1) / N)^{t}$.

\subsection{Secure Computation and NISC}

We use standard definitions of (composable) secure two-party computation. Our protocols can be analyzed and used either in a standalone setting, as formalized in [19, 31], or in a UC setting [20,38, 49]. It will be convenient to cast our protocols in a hybrid model that allows parallel calls to an ideal oblivious transfer functionality. These calls can be instantiated by any composable OT protocol (e.g., the "PVW protocol" [49] when considering UC security against malicious adversaries in the CRS model). We use $\lambda$ to denote a computational security parameter, which we view as a public parameter that is available to all algorithms even when not explicitly stated.

We will specifically be interested in 2-round protocols for "senderreceiver functionalities" that take an input $x$ from a receiver $\mathrm{R}$ and input $y$ from a sender $\mathrm{S}$, and deliver an output $f(x, y)$ to $\mathrm{R}$. The communication consists of a single message from the receiver to the sender followed by a single message from the receiver to the sender. Such protocols can be viewed as being non-interactive in that the receiver can publish its message $\hat{x}$ (which depends only on its input $x$ ) and then go offline, before even knowing who the sender will be. Then $\hat{x}$ can be used by any sender S (in fact, in some cases even multiple senders) by sending the encrypted output $\hat{z}$ to the receiver's mailbox. We use the term non-interactive secure computation from [38] (NISC for short) to highlight this qualitative advantage. When described in the OT-hybrid model, NISC protocols involve only one round of parallel OT calls. They can additionally involve a message from $\mathrm{R}$ to $\mathrm{S}$ and a message from $\mathrm{S}$ to $\mathrm{R}$, as long as these messages (in an honest execution) do not depend on outputs of the OT oracle. Such NISC protocols in the OT-hybrid model can be converted into NISC protocols in the plain model (or CRS model for malicious security) using any 2-round (parallel-)OT protocol.

\subsection{Pseudorandom Correlation Generators}

A (two-party) pseudorandom correlation generator (PCG) securely generates long correlated pseudo-randomness from a pair of correlated keys. Defining a PCG requires care, since the natural simulationbased definition is not realizable. Instead, the following relaxed definition has been proposed in $[15,16]$.

The ideal output distribution of a PCG is specified by a (long) target correlation $\left(R_{0}, R_{1}\right)$, e.g., $n$ independent instances of an OT correlation. This target correlation is specified by PPT algorithm $C$, called a correlation generator, where $C\left(1^{\lambda}\right)$ outputs a pair of strings. We furthermore restrict $C$ to be reverse-samplable in the following sense: there exists a PPT algorithm RSample such that for $\sigma \in\{0,1\}$, the correlation obtained via:

$$
\left\{\left(R_{0}^{\prime}, R_{1}^{\prime}\right) \mid\left(R_{0}, R_{1}\right) \stackrel{\$}{\leftarrow} C\left(1^{\lambda}\right), R_{\sigma}^{\prime}:=R_{\sigma}, R_{1-\sigma}^{\prime} \stackrel{\$}{\leftarrow} \operatorname{RSample}\left(\sigma, R_{\sigma}\right)\right\}
$$

is computationally indistinguishable from $C\left(1^{\lambda}\right)$.

Examples for standard and useful correlations, all of which are reverse-samplable, include Oblivious Transfer (OT) correlation, where $R_{0}$ includes $n$ independent pairs of bit-strings $\left(s_{0}^{i}, s_{1}^{i}\right)$ and $R_{1}$ includes $\left(c_{i}, s_{c_{i}}^{i}\right.$ for random bits $c_{i}$, and Vector-OLE (VOLE) correlation over a finite field $\mathbb{F}$, where $R_{0}=(\vec{u}, \vec{v})$ for random $\vec{u}, \vec{v} \in \mathbb{F}^{n}$, and $R_{1}=(x, \vec{u} x+\vec{v})$ for random $x \in \mathbb{F}$.

Definition 2.4 (Pseudorandom Correlation Generator (PCG) [16]). Let $C$ be a reverse-samplable correlation generator. A pseudorandom correlation generator (PCG) for $C$ is a pair of algorithms (PCG.Gen, PCG.Expand) with the following syntax:

- PCG.Gen $\left(1^{\lambda}\right)$ is a PPT algorithm that given a security parameter $\lambda$, outputs a pair of seeds $\left(\mathrm{k}_{0}, \mathrm{k}_{1}\right)$;

- PCG.Expand $\left(\sigma, \mathrm{k}_{\sigma}\right)$ is a polynomial-time algorithm that given party index $\sigma \in\{0,1\}$ and a seed $\mathrm{k}_{\sigma}$, outputs a bit string $R_{\sigma} \in\{0,1\}^{n}$.

The algorithms (PCG.Gen, PCG.Expand) should satisfy:

- Correctness. The correlation obtained via:

$$
\begin{gathered}
\left\{\left(R_{0}, R_{1}\right) \mid\left(\mathrm{k}_{0}, \mathrm{k}_{1}\right) \stackrel{\$}{\leftarrow} \text { PCG.Gen }\left(1^{\lambda}\right), R_{\sigma} \leftarrow \text { PCG.Expand }\left(\sigma, \mathrm{k}_{\sigma}\right)\right\} \\
\text { is computationally indistinguishable from } C\left(1^{\lambda}\right) .
\end{gathered}
$$

- Security. For corrupted party $\sigma \in\{0,1\}$, the following two distributions are computationally indistinguishable:

$$
\begin{aligned}
& \left\{\left(\mathrm{k}_{\sigma}, R_{\bar{\sigma}}\right) \mid\left(\mathrm{k}_{0}, \mathrm{k}_{1}\right) \stackrel{\$}{\leftarrow} \mathrm{PCG} . G e n\left(1^{\lambda}\right), R_{\bar{\sigma}} \leftarrow \text { PCG.Expand }\left(\bar{\sigma}, \mathrm{k}_{\bar{\sigma}}\right)\right\} \text { and } \\
& \left\{\left(\mathrm{k}_{\sigma}, R_{\bar{\sigma}}\right) \mid\left(\mathrm{k}_{0}, \mathrm{k}_{1}\right) \stackrel{\$}{\leftarrow} \operatorname{PCG} . G e n\left(1^{\lambda}\right), R_{\sigma} \leftarrow \operatorname{PCG} . \operatorname{Expand}\left(\sigma, \mathrm{k}_{\sigma}\right),\right. \\
& \left.R_{\bar{\sigma}} \stackrel{\$}{\leftarrow} \operatorname{RSample}\left(\sigma, R_{\sigma}\right)\right\}
\end{aligned}
$$

where $\bar{\sigma}=1-\sigma$ and RSample is the reverse sampling algorithm for $C$.

As shown in [16], a PCG as defined above can be used as a dropin replacement' for ideal correlated randomness generated by $C$ in any application that remains secure even when $C$ is replaced by the following corruptible version $\tilde{C}$. In $\tilde{C}$ the corrupted party can choose its own randomness, and the randomness of the honest party $R_{1-\sigma}$ is obtained by applying RSample. It turns out that in most concretely efficient MPC protocols that consume correlated randomness, security still holds even with this corruptible variant. In particular, this holds for the simple protocols that implement standard (chosen-input) OT or VOLE from the corresponding correlations. However, applying PCGs, the pair of keys $\left(\mathrm{k}_{0}, \mathrm{k}_{1}\right)$ to be generated either by a trusted dealer or by a secure protocol realizing PCG.Gen.

\section{PCG PROTOCOLS AND SILENT NISC}

We now define two new cryptographic primitives we introduce in this work: A pseudorandom correlation generation protocol (PCG protocol for short) and a non-interactive secure computation protocol with silent preprocessing (silent NISC for short).

\subsection{PCG Protocols}

The above notion of PCG gives a deterministic procedure for securely generating long sources of correlated randomness from short but suitably correlated seeds. It does not explicitly address the question of generating the seeds. In the following we formalize a natural generalization of PCGs to a low-communication protocol for securely generating long sources of correlated randomnness from 
scratch. By "low communication" we means that the total communication complexity is sublinear in the output length. ${ }^{3}$

We take the following natural definition approach: a PCG protocol for an ideal correlation $C$ is a secure two-party protocol (in the usual sense) for the corruptible correlated randomness functionality $\tilde{C}$ described below.

Definition 3.1 (PCG protocol). Let $C$ be a reverse-samplable correlation generator. Define a randomized functionality $\tilde{C}$ that takes from a corrupted party $\sigma$ a string $\tilde{r}_{\sigma}$ as input, and outputs to the honest party $\bar{\sigma}$ a string $r_{\bar{\sigma}}$ sampled by $\operatorname{RSample}\left(\sigma, \tilde{r}_{\sigma}\right)$. If no party is corrupted, $\tilde{C}$ outputs to both parties a fresh pair of outputs generated by $C$. A (two-party) PCG protocol is a two-party protocol realizing $\tilde{C}$ in which the communication complexity grows sublinearly with the output length. In the case of security against semi-honest adversaries, we still allow the ideal-model corrupted party (if any) to pick its input $\tilde{r}_{\sigma}$ for $\tilde{C}$ arbitrarily, whereas the real-model adversary must follow the protocol.

As a simple corollary of an MPC composition theorem, a PCG protocol for $C$ can serve as a substitute for ideal correlated randomness $C$ in any higher-level application that remains secure even when $C$ is replaced by $\tilde{C}$. Indeed, this is the case for standard MPC protocols that rely on OT correlations or other types of simple correlations, both for semi-honest and malicious security.

A general way of obtaining a PCG protocol is by distributing the randomized key generation functionality PCG.Gen of a PCG (as in Definition 2.4) via a secure two-party computation protocol, and then locally applying PCG.Expand. Indeed, this is the approach suggested in [16] for the purpose of applying PCGs in the context of "MPC with silent preprocessing." However, our notion of a PCG protocol is less stringent than an alternative definition that requires securely emulating PCG.Gen for some PCG, while at the same time being as good for applications. We make use of this extra degree of freedom in our PCG protocols for the malicious model.

A central contribution of this work is the construction of tworound $\mathrm{PCG}$ protocols, namely ones involving only a message from $\mathrm{R}$ to $\mathrm{S}$ followed by a message from $\mathrm{S}$ to $\mathrm{R}$. We refer to such a protocol as a non-interactive PCG protocol. We use the following syntax to highlight the fact that the message of $\mathrm{R}$ can be published as a "public key" before the sender(s) are known.

Definition 3.2 (Non-interactive PCG protocol). A non-interactive $P C G$ protocol is defined by 4 algorithms with the following syntax:

- R.Gen $\left(1^{\lambda}\right) \rightarrow\left(s k_{\mathrm{R}}, p k_{\mathrm{R}}\right)$

- $\operatorname{S.Gen}\left(p k_{\mathrm{R}}\right) \rightarrow\left(s k_{\mathrm{S}}, m_{\mathrm{S}}\right)$

- R.Expand $\left(s k_{\mathrm{R}}, m_{\mathrm{S}}\right) \rightarrow r_{\mathrm{R}}$

- S.Expand $\left(s k_{\mathrm{S}}\right) \rightarrow r_{\mathrm{S}}$

We say that the above algorithms define a non-interactive PCG protocol for a reverse-samplable correlation $C$ if the two-round protocol they naturally define (where each party outputs the output of Expand) is a PCG protocol for $C$ as in Definition 3.1.

In a non-interactive PCG protocol as above, the two Gen algorithms can be viewed as defining a cheap setup that results in short,

\footnotetext{
${ }^{3}$ In fact, in all protocols presented in this paper, the communication complexity only grows polylogarithmically with the output length, under widely believed variants of the LPN assumption.
}

correlated keys. The two Expand algorithms are used to locally perform "silent preprocessing" that generates useful correlated randomness (e.g., many instances of an OT correlation, or few instances of a long VOLE correlation). In the most useful special case of OT correlations, we will refer to a non-interactive PCG that makes a small number of parallel OT calls as a non-interactive (or 2-round) silent OT extension protocol.

\subsection{Silent NISC}

In this section we define our new notion of non-interactive secure computation with silent preprocessing, or silent NISC for short. A silent NISC protocol for $f$ can be viewed as a "best-of-both-worlds" combination of a non-interactive PCG protocol (see Definition 3.2) and a NISC protocol (see Section 2.3). That is, a 2-round (choseninput) secure computation protocol that supports "silent preprocessing" followed by a light-weight (and often "non-cryptographic") online phase, without additional interaction.

Combining non-interactive PCG and NISC protocols in a generic way does not achieve the above goal, since it involves 4 rounds: two to generate the correlated randomness, and two to use it. To collapse these 4 rounds into two, we rely on the following feature of our concrete non-interactive PCG constructions. For useful NISC correlations such as OT and VOLE, the receiver's piece of the correlated randomness $r_{\mathrm{R}}$ can be split into two parts: $r_{\mathrm{R}}^{\text {in }}$, which is used to mask its input, and $r_{\mathrm{R}}^{\text {out }}$, used to unmask the output. The key feature is that the construction allows $\mathrm{R}$ to locally generate $r_{\mathrm{R}}^{\text {in }}$ from its public key $p k_{\mathrm{R}}$ alone, independently of the sender. This enables $\mathrm{R}$ to prepare to a future NISC before the sender is even known.

More concretely, let $f(x, y)$ be a sender-receiver functionality with receiver input $x$ and sender input $y$. Useful examples for which we get efficient solutions include: (1) $n$ instances of string-OT; (2) bitwise-AND of two $n$-bit strings; (3) inner product of two length- $n$ vectors over $\mathbb{F}$; (4) a general function $f$ represented by a Boolean circuit, which can be efficiently and non-interactively reduced to (1) via garbled circuits (see $[1,38,46]$ for such black-box reductions for the malicious model).

A NISC protocol with silent preprocessing (or silent NISC) for $f$ is defined by 8 algorithms:

- R.Gen $\left(1^{\lambda}\right) \rightarrow\left(s k_{\mathrm{R}}, p k_{\mathrm{R}}\right)$

- R.Expand ${ }^{\text {in }}\left(s k_{\mathrm{R}}\right) \rightarrow r_{\mathrm{R}}^{\text {in }}$

- $\operatorname{S.Gen}\left(p k_{\mathrm{R}}\right) \rightarrow\left(s k_{\mathrm{S}}, p k_{\mathrm{S}}\right)$

- R.Expand ${ }^{\text {out }}\left(s k_{\mathrm{R}}, p k_{\mathrm{S}}\right) \rightarrow r_{\mathrm{R}}^{\text {out }}$

- S.Expand $\left(s k_{\mathrm{S}}\right) \rightarrow r_{\mathrm{S}}$

- $\operatorname{R} . \operatorname{Msg}\left(r_{\mathrm{R}}^{\mathrm{in}}, x\right) \rightarrow \hat{x}$

- $\operatorname{S.Msg}\left(r_{\mathrm{S}}, \hat{x}, y\right) \rightarrow \hat{z}$

- R.Dec $\left(r_{\mathrm{R}}^{\text {out }}, x, \hat{z}\right) \rightarrow z$

The security requirement is that the 2-round protocol obtained by executing the above algorithms in any consistent order satisfies the security requirement of a (standard) NISC protocol for $f$.

To clarify the intended use and the features of our model for noninteractive secure computation protocols with silent preprocessing, we provide on Figure 2 a pictural representation of the protocol flow, illustrating the interdependencies between the algorithms, and we identify the main features of each of the algorithms (whether they require small communication, or only silent computation; whether they require cryptographic or non-cryptographic computation). 


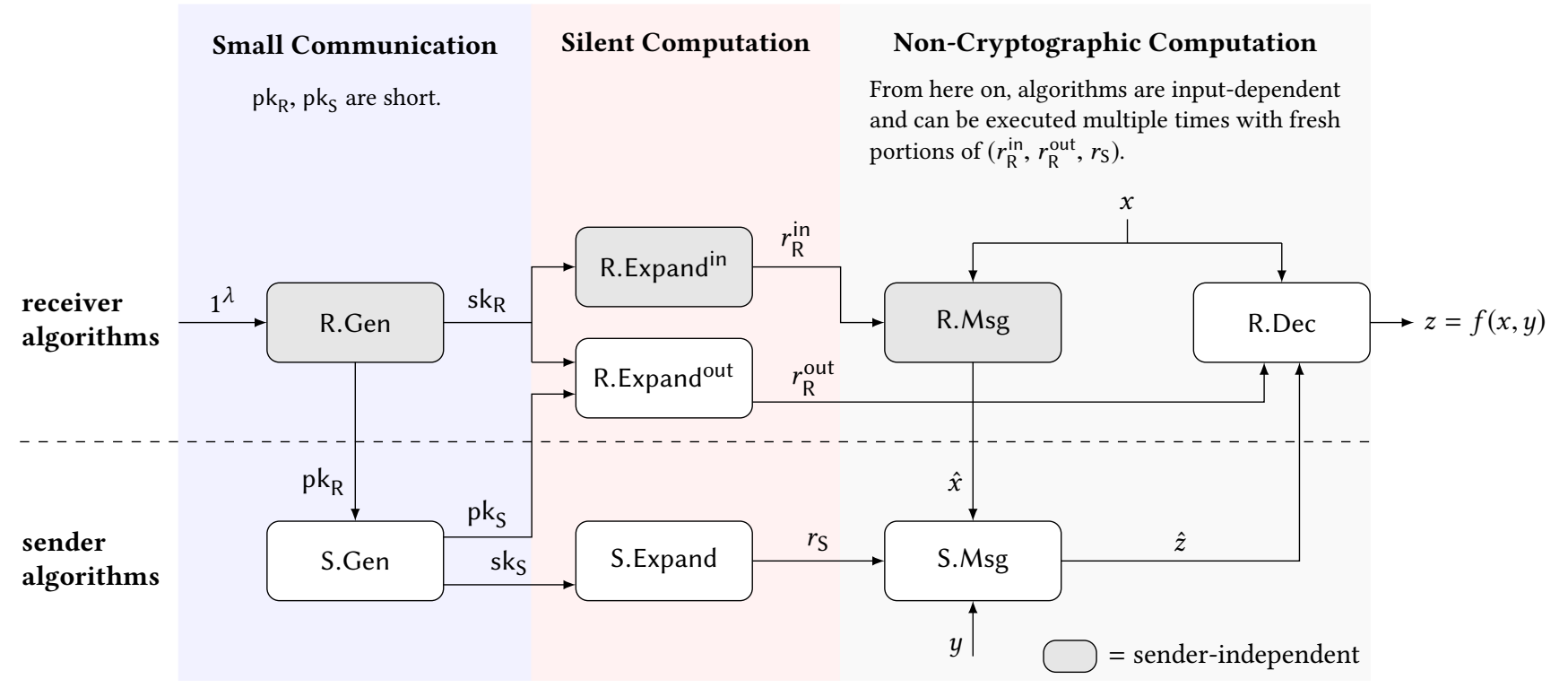

Figure 2: Pictural representation of the protocol flow for non-interactive secure computation with silent preprocessing. The receiver input is $y$, the sender input is $x$, and the target output is $z=f(x, y)$.

The 3 Expand algorithms define the "silent preprocessing" phase, that can be executed before the inputs are known. The last 3 algorithms define the online part of the NISC protocol, which is carried out once the inputs are known. Among the four examples given above, this part is "non-cryptographic" in the first three cases, and makes a black-box use of symmetric crypto in the last one.

We will be particularly interested in silent NISC realizing many parallel OTs using few parallel OTs, which can be viewed as a noninteractive, chosen-input variant of silent OT extension. While here one cannot make the communication complexity sublinear in the input length, our goal (which we achieve both in theorem and in practice) to make the communication very close to the total input length. This is the case even for the more challenging case of 1-bit OT, for which standard OT extension techniques that make a blackbox use of cryptography $[8,37,41,44]$ have a high communication overhead compared to the input length.

\section{OPTIMIZED VOLE/OT CONSTRUCTION}

\subsection{Simplified subfield VOLE generator}

We provide a construction of a PCG for subfield-VOLE correlations on Fig. 3. Recall that in subfield-VOLE, one party receives random vectors $\vec{u} \in \mathbb{F}_{p}^{N}$ and $\vec{v} \in \mathbb{F}_{p^{r}}^{N}$, while the other party gets a random $x \in \mathbb{F}_{p^{r}}$, and $\vec{w}=\vec{u} x+\vec{v}$. The construction follows the informal description from Section 1.2 (where we described the special case $p=2$, which is equivalent to correlated OT), and is essentially the same as the construction in [16], with a puncturable PRF instead of a DPF. Likewise, the security analysis is essentially identical to the analysis of [16].

\subsection{Instantiating the puncturable PRF}

We use a simple puncturable PRF based on the GGM approach [32] (as defined in Section 2). To build a PPRF supporting $t$ punctured points, we simply create $t$ independent GGM PRFs, each punctured once. Evaluation of the final PPRF is defined by adding the evaluations of all $t$ GGM-based PRFs.

More Efficient Puncturing Strategy. The key size for the above $t$-puncturable PRF is $t \cdot \lambda \log (N)$. It is possible to reduce this size to $t \cdot \lambda \log (N / t)$ with a more optimized puncturing strategy; however, this alternative construction is not compatible with our optimized distributed generation protocols of Section 5 and Section 6. It is nonetheless useful in a setting where a trusted dealer is available to distribute the PCG seeds, or where computation is not a bottleneck compared to long-term storage. For a formal treatment we refer to the full version.

\section{SEMI-HONEST PCG PROTOCOL AND TWO-ROUND OT EXTENSION}

In this section, we show how to securely compute the Gen algorithm from Fig. 3, in just 2 rounds (assuming any 2-round OT). Using the construction of [16], this leads to a distributed protocol for generating random OT correlations as well, assuming in addition a correlation-robust hash function. Then, we observe that our protocols satisfy a specific feature, which allows them to be derandomized into chosen-input VOLEs and OTs, without increasing their round complexity; this leads to 2-round OT extension and VOLE extension protocols, with silent preprocessing. Our construction relies on the GGM puncturable PRF [32] constructed from any length-doubling pseudorandom generator $G$.

On VOLE and reverse VOLE. In a typical (chosen-input) VOLE, the sender inputs $(\vec{u}, \vec{v})$, while the receiver inputs $x$ and gets $\vec{w}=\vec{u} x+\vec{v}$. 


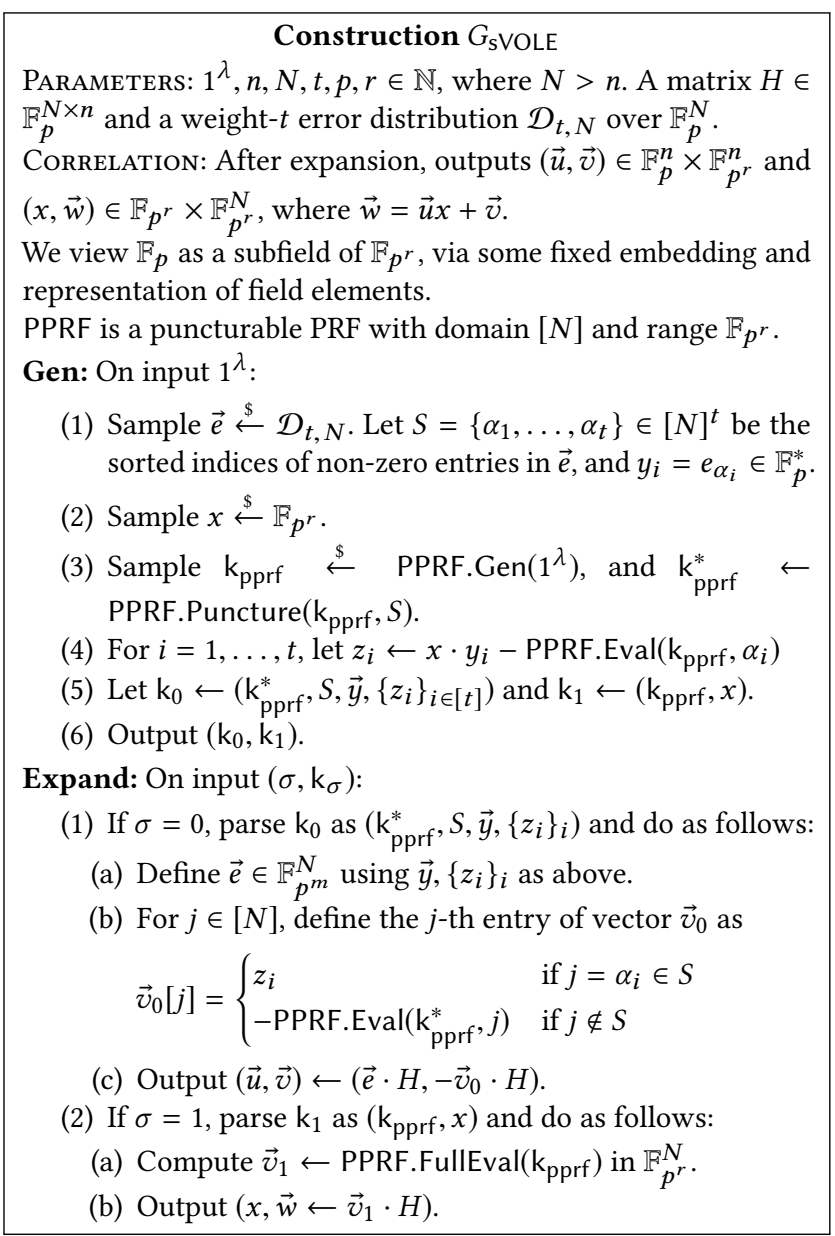

Figure 3: PPRF-based PCG for subfield vector-OLE

In our two-round protocols, we actually obtain a slightly different variant, called reverse VOLE [4], where the sender inputs $x, \vec{w}$, while the receiver inputs $\vec{u}$ and learns $\vec{v}=\vec{w}-\vec{u} x$.

\subsection{Distributed GGM-PPRF Correlation}

We first consider a functionality where a party $\mathrm{R}$ holds a PPRF key $k_{\text {pprf }} \in\{0,1\}^{\lambda}$ for the GGM PPRF [32], and a point $\alpha=$ $\alpha_{1} \cdots \alpha_{\ell}$ where $\ell=\ell(\lambda)$ is logarithmic in $\lambda$, and a party $\mathrm{S}$ holds a value $\beta \in\{0,1\}^{\lambda}$. The functionality computes and gives $k\{\alpha\}, \beta-$ PPRF.Eval $(k, \alpha)$ to R. The functionality is represented on Figure 4.

THEOREM 5.1. Assuming a black-box access to a PRG, there exists a 2-party protocol for $\mathcal{F}_{\mathrm{PPRF}-\mathrm{GGM}}$, with semi-honest security in the OThybrid model, and the following efficiency features. The computational complexity is dominated by $O\left(2^{\ell}\right)$ calls to a length-doubling $P R G$ $G:\{0,1\}^{\lambda} \mapsto\{0,1\}^{2 \lambda}$. The interaction consists of $\ell$ parallel calls to $\mathcal{F}_{\mathrm{OT}}$ and has communication complexity $\lambda+(3 \lambda+1) \ell$.

PROOF. We represent the protocol ПPPRF-GGM satisfying the requirements of Theorem 5.1 on Figure 5. At a high-level, our protocol proceeds as follows: at each level $i$ of the GGM tree, the holder of

\section{Functionality $\mathcal{F}_{\mathrm{PPRF}-\mathrm{GGM}}$ :}

PARAmeters: $1^{\lambda}, \ell, p, r \in \mathbb{N}$. PPRF is a puncturable PRF with domain $\{0,1\}^{\ell}$, key space $\{0,1\}^{\lambda}$, and range $\mathbb{F}_{p^{r}}$.

INPUTS:

- S inputs $\beta \in \mathbb{F}_{p^{r}}$ and a PPRF key $\mathrm{k}_{\text {pprf }} \in\{0,1\}^{\lambda}$.

- R inputs $\alpha \in\{0,1\}^{\ell}$.

FunCTIONALITY:

- Compute $\mathrm{k}_{\text {pprf }}^{*}=$ PPRF.Puncture $\left(\mathrm{k}_{\mathrm{pprf}}, \alpha\right)$.

- Send $\mathrm{k}_{\mathrm{pprf}}^{*}$ and $t=\beta$ - PPRF.Eval $\left(\mathrm{k}_{\mathrm{pprf}}, \alpha\right)$ to R.

Figure 4: Functionality for distributing a PPRF correlation

the PRF key k computes the XOR $i_{R}^{i}$ of all odd-numbered nodes, and the XOR $t_{L}^{i}$ of all even-numbered nodes. Using a single 1-out-of-2 OT, the receiver obtains one of $\left(t_{L}^{i}, t_{R}^{i}\right)$. The protocol maintains the invariant that at the level $i-1$, the receiver can compute (from the previously stored information) all node values except one, implying that at level $i$, the receiver can compute all node values except two; recovering one of $\left(t_{L}^{i}, t_{R}^{i}\right)$ allows him to compute exactly one of those two values, maintaining the invariant. At the end of the protocol, the receiver has stored $\ell$ intermediate keys $(\ell$ being the depth of the tree) which allow to compute all PRF outputs, except one. Transmitting a single additional value allow the sender to reveal him this value up to an offset $\beta$.

Correctness. We first show that the $s_{\alpha_{i}^{*}}^{i}$ values form a correct PRF key punctured at $\alpha$. We need that for each $i, s_{\alpha_{i}^{*}}^{i}$ equals the GGM tree value that is sibling to the unique node on level $i$ lying on the path to leaf $\alpha$. This clearly holds for the first level, $i=1$. On subsequent levels, $R$ first computes the $2^{i}-2$ values at level $i$ that it can obtain from the previous values it knows, and then uses these to compute the final missing value $s_{\alpha_{i}^{*}}^{i}$. It does this by XORing (resp. summing over $\mathbb{F}_{p^{r}}$, for the last level) with the $i$-th OT output all-but-one of the odd-indexed, or even-indexed, values, depending on the choice $\overline{\alpha_{i}}$. Since the sender's OT inputs contain the XOR (resp. sum over $\mathbb{F}_{p^{r}}$, for the last level) of every odd- or even-indexed value, the receiver ends up with the value of the sibling node to $s_{\alpha_{i}}^{i}$. To see correctness of the final correction value $t$, a similar reasoning as above shows that $t=\beta-\operatorname{PPRF} \operatorname{Eval}(k, \alpha)$, as required.

Security. We exhibit a simulator Sim that generates a view indistinguishable from an honest run of the protocol as long as a single party is corrupted.

Case 1: $S$ is corrupted. The simulation of $R$ is straightforward, since $R$ does not send any message directly to $S$ in the protocol; $R$ only send inputs to $\mathcal{F}_{\text {OT }}$.

Case 2: $R$ is corrupted. Sim receives the input $\alpha$ and the target output $\left(\left\{s_{\alpha_{i}^{*}}^{i}\right\}_{i \in[\ell]}, t\right)$ of $R$. Sim defines $t^{1} \cdots t^{\ell}$ and the values $\left(s_{j}^{i}\right)_{i, j}$ inductively, starting with $t^{1} \leftarrow s_{\overline{\alpha_{1}}}^{1}$ and following the output procedure of $R$ (see Figure 5). Eventually, Sim computes

$$
c^{\prime}=t-\left(t^{\ell}+\sum_{j=0, j \neq \alpha}^{2^{\ell-1}} s_{2 j+\alpha_{\ell}}^{\ell}\right) .
$$


Then, Sim simulates the OT sender using input $\left(t^{i}, d_{i}\right)$ as input if $\overline{\alpha_{i}}=0$, and $\left(d_{i}, t^{i}\right)$ as input otherwise, where $d_{i}$ is an arbitrary dummy value; Sim also sends $c^{\prime}$ in parallel to the OTs. The indistinguishability of the simulation follows directly from the definition of $\mathcal{F}_{\mathrm{OT}}$ and by construction of $c^{\prime}$.

\subsection{Semi-Honest Non-Interactive PCG Protocol for Subfield-VOLE Correlations}

We now explain how to implement a semi-honest public-key PCG for the subfield-VOLE correlation in the $\mathcal{F}_{\text {PPRF-GGM-hybrid model, }}$ by describing a 2-message 2-party semi-honest protocol to distributively execute the procedure $G_{\mathrm{S} V O L E}$.Gen. The functionality $\mathcal{F}_{\mathrm{Gen}}$ is represented on Figure 6 . When $p>2$, the implementation requires in addition a single (subfield-) reverse vector-OLE on vectors of length $t$. Reverse vector-OLE can be implemented in two rounds under an appropriate variant of LPN [4] or using linearly homomorphic encryption. We represent the functionality $\mathcal{F}_{\text {rev-VOLE }}$ on Figure 7. Note that in a reverse vector-OLE protocol, the sender is the one holding the input $x$ (while in a standard vector-OLE, $x$ is held by the receiver).

THEOREM 5.2. There exists a 2-message protocol $\Pi_{\text {sVOLE-Gen }}$ which realizes the functionality $\mathcal{F}_{\mathrm{SVOLE}}$-Gen $\left(1^{\lambda}, N, t, p, r\right)$, with semi-honest security in the ( $\left.\mathcal{F}_{\text {PPRF-GGM }}, \mathcal{F}_{\text {rev-VOLE }}\right)$-hybrid model, using $t$ calls to $\mathcal{F}_{\mathrm{PPRF}-\mathrm{GGM}}$, a single call to $\mathcal{F}_{\text {rev-VOLE }}(t, p)$, and no further communication. Furthermore, when $p=2$, the functionality can be implemented directly using $t$ calls to $\mathcal{F}_{\text {PPRF-GGM }}$, and no call to $\mathcal{F}_{\text {rev-VOLE }}$

We present the protocol $\Pi_{\text {sVOLE-Gen }}$ in Figure 8. Correctness follows easily by inspection: for $i=1$ to $t$, we have $z_{i}=w_{i}+$ $c_{i}=\left(b_{i}-\right.$ PPRF.Eval $\left.\left(\mathrm{k}_{\mathrm{pprf}}, \alpha_{i}\right)\right)+c_{i}=x \cdot y_{i}-\mathrm{PPRF} . \operatorname{Eval}\left(\mathrm{k}_{\mathrm{pprf}}, \alpha_{i}\right)$. Security is straightforward. We note that when $p=2$, since $\vec{y}$ is a weight- $t$ vector, it always hold that $y_{i}=1$, hence computing a share of $x \cdot y_{i}=x$ is trivial and does not require a call to the VOLE functionality.

Implementing $\mathcal{F}_{\text {PPRF-GGM }}$ with the protocol $\Pi_{\text {PPRF-GGM }}$ and $\mathcal{F}_{\text {OT }}$ with any 2-round semi-honest OT protocol, this immediately leads to a semi-honest non-interactive PCG protocol $\Pi_{\text {sVOLE }}\left(\mathbb{F}_{q}\right)$ for the subfield-VOLE correlation:

- R.Gen $\left(1^{\lambda}\right)$ : sets $p k_{R}$ to be the first message of $\Pi_{\text {sVOLE-Gen }}$ and $s k_{R}$ to be the secret state of $R$.

- S.Gen $\left(\mathrm{pk}_{\mathrm{R}}\right)$ : sets $m_{\mathrm{S}}$ to be the second message of $\Pi_{\mathrm{sVOLE}}$-Gen on first message $p k_{R}$, and $s k_{S}$ to be the sender output in $\Pi_{\text {sVOLE-Gen. }}$

- R.Expand $\left(\mathrm{sk}_{\mathrm{R}}, m_{\mathrm{S}}\right)$ : computes the output $\mathrm{k}_{0}$ of the receiver from the state $s k_{R}$ and the second message $m_{\mathrm{S}}$, and outputs $G_{\text {SVOLE. }}$ Expand $\left(0, \mathrm{k}_{0}\right)$.

- S.Expand(sks) : outputs $G_{\mathrm{S} V O L E}$. Expand(1, sks).

Corollary 5.3. Assuming the $\left(\mathcal{H} W_{t}, H, \mathbb{F}_{q}\right)$-dual-LPN $\left(n^{\prime}, n\right)$ assumption, $\Pi_{\mathrm{s} V O L E-G e n}$ is a semi-honest non-interactive PCG protocol for subfield-VOLE correlations over an arbitrary extension field $\mathbb{F}_{q}$ of $\mathbb{F}_{2}$, which only makes a black-box use of a 1-out-of-2 semi-honest 2-message OT and a length-doubling PRG $G:\{0,1\}^{\lambda} \rightarrow\{0,1\}^{2 \lambda}$. By making additionally a single black-box use of a 2-message length- $t$ semi-honest reverse VOLE, this can be generalized to arbitrary fields.

\section{Protocol ПPPRF-GGM:}

PARAmeters: $1^{\lambda}, \ell, p, r \in \mathbb{N}$. PPRF is the GGM puncturable PRF with domain $\{0,1\}^{\ell}$, key space $\{0,1\}^{\lambda}$, and range $\mathbb{F}_{p^{r}}$, constructed from a length-doubling PRG $G:\{0,1\}^{\lambda} \mapsto\{0,1\}^{2 \lambda}$, and a second PRG $G^{\prime}:\{0,1\}^{\lambda} \mapsto\left(\mathbb{F}_{p^{r}}\right)^{2}$ used to compute the PRF outputs on the last level of the tree.

INPUTS:

- R inputs $\alpha \in\{0,1\}^{\ell}$.

- S inputs $\beta \in \mathbb{F}_{p^{r}}$ and a PPRF key $\mathrm{k}_{\text {pprf }} \in\{0,1\}^{\lambda}$.

Protocol:

(1) R and $\mathrm{S}$ execute in parallel $\ell$ calls to $\mathcal{F}_{\mathrm{OT}}$, where for $i=1$ to $\ell-1$ :

- $\mathrm{R}$ uses as input the choice bit $\overline{\alpha_{i}}$;

- S computes the $2^{i}$ partial evaluations at level $i$ of the GGM tree defined by $k$, denoted $s_{0}^{i}, \ldots, s_{2^{i}-1}^{i}$ (in leftto-right order) and uses the two OT inputs

$$
t_{L}^{i}=\bigoplus_{j \in\left[0,2^{i-1}\right)} s_{2 j}^{i}, \quad t_{R}^{i}=\bigoplus_{j \in\left[0,2^{i-1}\right)} s_{2 j+1}^{i} .
$$

and for the last OT,

- R uses as input the choice bit $\overline{\alpha_{\ell}}$;

- S computes the $2^{\ell}$ evaluations of the GGM tree defined by $k$, denoted $s_{0}^{\ell}, \ldots, s_{2^{i}-1}^{\ell} \in\left(\mathbb{F}_{p^{r}}\right)^{2^{\ell}}$ (in left-to-right order) and uses the two OT inputs

$$
t_{L}^{\ell}=\sum_{j=0}^{2^{\ell-1}} s_{2 j}^{\ell}, \quad t_{R}^{\ell}=\sum_{j=0}^{2^{\ell-1}} s_{2 j+1}^{\ell} .
$$

(2) In parallel to the OT calls, S sends $c=\beta-\left(t_{L}^{\ell}+t_{R}^{\ell}\right)$ to R. OutPut: $\mathrm{R}$ computes its output as follows:

(1) Let $t^{1}$ be R's output in the first OT. Define $s_{\overline{\alpha_{1}}}^{1}=t^{1}$.

(2) For $i=2, \ldots, \ell-1$ :

(a) Compute $\left(s_{2 j}^{i}, s_{2 j+1}^{i}\right)=G\left(s_{j}^{i-1}\right)$, for $j \in$ $\left[0, \ldots, 2^{i-1}\right), j \neq \alpha_{1} \cdots \alpha_{i-1}$.

(b) Let $t^{i}$ be the output from the $i$-th OT.

(c) Define $\alpha_{i}^{*}=\alpha_{1} \cdots \alpha_{i-1} \overline{\alpha_{i}}$. Compute

$$
s_{\alpha_{i}^{*}}^{i}=t^{i} \oplus \bigoplus_{\substack{j \in\left[0,2^{i-1}\right), j \neq \alpha_{i}^{*}}} s_{2 j+\overline{\alpha_{i}}}^{i}
$$

(3) Compute $\left(s_{2 j}^{\ell}, s_{2 j+1}^{\ell}\right)=G^{\prime}\left(s_{j}^{i-1}\right)$, for $j \in\left[0, \ldots, 2^{\ell-1}\right), j \neq$ $\alpha_{1} \cdots \alpha_{\ell-1}$.

(4) $\mathrm{R}$ receives $c$, and computes

$$
t=c+t^{\ell}+\sum_{j=0, j \neq \alpha}^{2^{\ell-1}} s_{2 j+\alpha_{\ell}}^{\ell}
$$

(5) R outputs the punctured key $\left\{s_{\alpha_{i}^{*}}^{i}\right\}_{i \in[\ell]}$, and the final correction value $t$.

Figure 5: Protocol ПPPRF-GGM for distributing a GGM-based PPRF correlation with semi-honest security in the $\mathcal{F}_{\mathrm{OT}}-$ hybrid model 


\section{Functionality $\mathcal{F}_{\text {sVOLE-Gen }}$}

Parameters: $1^{\lambda}, N, t, p, r \in \mathbb{N}$. PPRF is a puncturable PRF with domain $[N]$, key space $\{0,1\}^{\lambda}$, and range $\mathbb{F}_{p^{r}}$.

INPUTS:

- $\mathrm{R}$ inputs a weight- $t$ vector $\vec{e} \in \mathbb{F}_{p}^{N}$. Let $\mathcal{S}=$ $\left\{\alpha_{1}, \ldots, \alpha_{t}\right\} \in[N]^{t}$ be the sorted indices of non-zero entries in $\vec{e}$, and $y_{i}=e_{\alpha_{i}} \in \mathbb{F}_{p}^{*}$.

- S inputs $x \in \mathbb{F}_{p^{r}}$ and a PPRF key kprf $\in\{0,1\}^{\lambda}$.

FunCTIONALITY:

(1) Compute $\mathrm{k}_{\text {pprf }}^{*} \leftarrow$ PPRF.Puncture $\left(\mathrm{k}_{\text {pprf }}, \mathcal{S}\right)$.

(2) For $i=1, \ldots, t$, let $z_{i} \leftarrow x \cdot y_{i}-\operatorname{PPRF} . \operatorname{Eval}\left(\mathrm{k}_{\mathrm{pprf}}, \alpha_{i}\right)$

(3) Let $\mathrm{k}_{0} \leftarrow\left(\mathrm{k}_{\mathrm{pprf}}^{*}, \mathcal{S}, \vec{y},\left\{z_{i}\right\}_{i \in[t]}\right)$ and $\mathrm{k}_{1} \leftarrow\left(\mathrm{k}_{\mathrm{pprf}}, x\right)$.

(4) Output $k_{0}$ to $R$ and $k_{1}$ to $S$.

Figure 6: Functionality for the Generation Procedure of the Subfield-VOLE Generator

Functionality $\mathcal{F}_{\text {rev-VOLE }}$
PARAmETERs: $t, p, r \in \mathbb{N}$.
InPUT: The sender S inputs a pair $(\vec{b}, x) \in \mathbb{F}_{p^{r}}^{t} \times \mathbb{F}_{p^{r}}$. The receiver
R inputs a vector $\vec{y} \in \mathbb{F}_{p}$.
FunCtionAlity: Compute $\vec{c} \leftarrow \vec{y} x-\vec{b}$ and output $\vec{c}$ to R.

Figure 7: Reverse Vector-OLE Functionality over a Field $\mathbb{F}_{p}$

In the above corollary, $\Pi_{\text {sVOLE-Gen }}$ makes $t \cdot\left\lceil\log n^{\prime}\right\rceil$ black-box accesses to the 1-out-of- 2 semi-honest 2-message OT, $t \cdot n^{\prime}$ black-box accesses to a length-doubling PRG, and additionally computes one matrix-vector multiplication with $H$. Regarding communication, the size of $\mathrm{pk}_{\mathrm{R}}$ is $t \cdot\left\lceil\log n^{\prime}\right\rceil \cdot N_{\mathrm{R}}$ and the size of $m_{\mathrm{S}}$ is $t \cdot\left(\lambda+\left\lceil\log n^{\prime}\right\rceil \cdot\right.$ $N_{\mathrm{S}}$ ), where $N_{\mathrm{R}}$ (resp. $N_{\mathrm{S}}$ ) denote the receiver communication (resp. the sender communication) in the underlying OT protocol; over general fields, there is an additional $+M_{\mathrm{R}}(t, q, r)$ term in the size of $\mathrm{pk}_{\mathrm{R}}$ and $+t \cdot M_{\mathrm{S}}(t, q, r)$ in the size of $m_{\mathrm{S}}$, where $M_{\mathrm{R}}(t, q, r)$ (resp. $\left.M_{\mathrm{S}}(t, q, r)\right)$ denote the receiver communication (resp. the sender communication) in the underlying length- $t$ reverse subfield-VOLE protocol over $\mathbb{F}_{q^{r}}$.

\subsection{Semi-Honest Non-Interactive Secure Computation with Silent Preprocessing}

While the non-interactive PCG protocols of the previous section are interesting in their own right, we observe that they satisfy the features outlined in Section 3.2, and therefore lead to 2-round protocols, and even silent NISC, for the OT and the VOLE functionalities.

5.3.1 Semi-Honest 2-Round OT with Silent Preprocessing. As observed in [16], a PCG for subfield-VOLE correlation together with a correlation-robust hash function lead to a PCG $G_{\mathrm{OT}}$ for the ROT correlation. For completeness, we recall the construction $G_{\mathrm{OT}}$ on Figure 9. Using our distributed generation algorithm (which can be implemented in two rounds given any 2-round OT and 2-round

\section{Protocol $\Pi_{\mathrm{S} V O L E-G e n}$}

PARAmeters: $1^{\lambda}, N=2^{\ell}, t, p, r \in \mathbb{N}$. PPRF is a puncturable PRF with domain $[N]$ and range $\mathbb{F}_{p^{r}}$.

INPUTS:

- $\mathrm{R}$ inputs a weight- $t$ vector $\vec{e} \in \mathbb{F}_{p}^{N}$. Let $\mathcal{S}=$ $\left\{\alpha_{1}, \ldots, \alpha_{t}\right\} \in[N]^{t}$ be the sorted indices of non-zero entries in $\vec{e}$, and $y_{i}=e_{\alpha_{i}} \in \mathbb{F}_{p}^{*}$.

- S inputs $x \in \mathbb{F}_{p^{r}}$ and a PPRF key kprf $\in\{0,1\}^{\lambda}$.

PROTOCOL:

(1) S picks $\vec{b} \stackrel{\$}{\leftarrow} \mathbb{F}_{p}^{t}$.

(2) $\mathrm{R}$ and $\mathrm{S}$ call $\mathcal{F}_{\text {rev-VOLE }}(t, p)$ on respective inputs $(\vec{y},(\vec{b}, x))$. $\mathrm{R}$ receives an output $\vec{c}$.

(3) For $i=1$ to $t, \mathrm{R}$ and $\mathrm{S}$ call $\mathcal{F}_{\mathrm{PPRF}-\mathrm{GGM}}\left(1^{\lambda}, \ell, p, r\right)$ on respective inputs $\alpha_{i}$ and $\left(b_{i}, \mathrm{k}_{\mathrm{pprf}}\right)$. R receives an output $\left(\mathrm{k}_{\mathrm{pprf}}^{*}, w_{i}\right)$.

(4) For $i=1$ to $t$, R computes $z_{i} \leftarrow w_{i}+c_{i}$. R outputs $\left(\mathrm{k}_{\mathrm{pprf}}^{*}, \mathcal{S}, \vec{y},\left\{z_{i}\right\}_{i \in[t]}\right)$ and $\mathrm{S}$ outputs $\left(\mathrm{k}_{\mathrm{pprf}}, x\right)$.

Figure 8: Protocol for the Generation Procedure of the Subfield-VOLE Generator

\section{Construction $G_{\mathrm{OT}}$}

PARAmeters:

- Security parameter $1^{\lambda}$, integers $n, q=p^{r}$.

- An $\mathbb{F}_{p}$-correlation-robust function $\mathrm{H}:\{0,1\}^{\lambda} \times \mathbb{F}_{q} \rightarrow$ $\{0,1\}^{\lambda}$.

- The subfield-VOLE PCG ( $G_{\text {SVOLE }}$.Gen, $G_{\text {SVOLE }}$.Expand)

CORRELATION:

Outputs $\left(R_{0}, R_{1}\right)=\left(\left\{\left(u_{i}, w_{i, u_{i}}\right)\right\}_{i \in[n]},\left\{w_{i, j}\right\}_{i \in[n], j \in[p]}\right)$, where $w_{i, j} \stackrel{\$}{\leftarrow}\{0,1\}^{\lambda}$ and $u_{i} \stackrel{\$}{\leftarrow}\{1, \ldots, p\}$, for $i \in[n], j \in[p]$.

Gen: On input $1^{\lambda}$, output $\left(\mathrm{k}_{0}, \mathrm{k}_{1}\right) \leftarrow G_{\text {SVOLE }} \cdot \operatorname{Gen}\left(1^{\lambda}, n, p, q\right)$.

EXPAND: On input $\left(\sigma, \mathrm{k}_{\sigma}\right)$ :

(1) If $\sigma=0$ : compute $\left(\vec{u}, \vec{v}^{\prime}\right) \leftarrow G_{\text {SVOLE }}$ Expand $\left(\sigma, \mathrm{k}_{\sigma}\right)$, where $\vec{u} \in \mathbb{F}_{p}^{n}, \vec{v}^{\prime} \in \mathbb{F}_{q}^{n}$. Compute

$$
v_{i} \leftarrow \mathrm{H}\left(i, v_{i}^{\prime}\right) \quad \text { for } i=1, \ldots, n
$$

and output $\left(u_{i}, v_{i}\right)$.

(2) If $\sigma=1$ : compute $\left(x, \vec{w}^{\prime}\right) \leftarrow G_{\text {sVOLE }}$.Expand $\left(\sigma, \mathrm{k}_{\sigma}\right)$, where $x \in \mathbb{F}_{q}, \vec{w}^{\prime} \in \mathbb{F}_{q}^{n}$. Compute $w_{i, j} \leftarrow \mathrm{H}\left(i, w_{i}^{\prime}-j \cdot x\right) \quad$ for $i=1, \ldots, n, \forall j \in \mathbb{F}_{p}$ and output $\left\{w_{i, j}\right\}_{i, j}$.

Figure 9: PCG for $n$ sets of 1-out-of- $p$ random OT

subfield-VOLE), together with the standard protocol for choseninput OT from ROT, directly leads to a 2-round OT extension protocol, which performs $n$ OTs on $s$-bit strings with communication $(2 s+1) \cdot n+o(n)$ (for any $s)$. 
Theorem 5.4. Assuming the $\left(\mathcal{H} \mathcal{W}_{t}, H, \mathbb{F}_{p}\right)$-dual-LPN $\left(n^{\prime}, n\right)$ assumption, $\Pi_{\mathrm{OT}}$ is a semi-honest 2-round OT extension with silent preprocessing for generating $n$ 1-out-of-2 OTs, which makes o(n) black-box uses of a 2-round semi-honest 1-out-of-2 OT, and $O(n)$ black-box uses to a length-doubling $P R G$ and an $\mathbb{F}_{p}$-correlation robust hash function.

Assuming further any 2-round semi-honest reverse VOLE, there is a 2-round OT extension with silent preprocessing for 1-out-of-p OT with comparable costs, using additionnally one black-box execution of a reverse-VOLE on length-o(n) inputs.

Proof. In the above theorem, $\Pi_{\mathrm{OT}}$ additionally requires the computation of one matrix-vector multiplication with $H$. It has total communication $(2 s+1) \cdot n+o(n)$, where $s$ is the bit-length of the sender messages. We represent the protocol for 2-round OT extension on Figure 10.

Correctness. By the correctness of $\Pi_{\mathrm{SVOLE}-G e n}$ and $G_{\mathrm{OT}}$, it holds that $v_{i}=w_{i, u_{i}}$ for $i=1$ to $n$. Therefore, $m_{i, s_{i}}^{\prime}-v_{i}=m_{i, s_{i}}+$ $w_{i, t_{i}-s_{i}}-w_{i, u_{i}}=m_{i, s_{i}}$ since $t_{i}-s_{i}=u_{i}$.

Security. We exhibit a simulator Sim that generates a view indistinguishable from an honest run of the protocol as long as a single party is corrupted.

Case 1: $S$ is corrupted. Sim simulates $R$ by constructing $(\vec{e}, \vec{u})$ honestly, participating to $\Pi_{\mathrm{sVOLE}}-\mathrm{Gen}$ as $R$ does (note that this does not require any input of $R$ ). Sim simulates $\vec{t}$ by sending $\vec{t}^{\prime} \stackrel{\$}{\leftarrow} \mathbb{F}_{p}^{n}$. Since $\Pi_{\text {SVOLE-Gen }}$ securely emulates $\mathbb{F}_{\text {Gen }}$, no information about $\vec{u}$

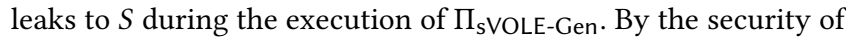
$G_{\mathrm{OT}}, \vec{u}$ is computationally indistinguishable from random from the viewpoint of $S$, hence so is $\vec{t}=\vec{u}+\vec{s}$; therefore, the simulation is indistinguishable from an honest run of the protocol.

Case 2: $R$ is corrupted. Sim receives $R$ 's input $\left(s_{i}\right)_{i \leq n}, R$ 's random tape, and the corresponding target output $\left(m_{i, s_{i}}\right)_{i}$ from the OT functionality. Sim simulates $S$ by sampling $\mathrm{k}_{1}$ and computing the $w_{i, j}$ honestly (this does not require the input of $S$ ). Sim computes the random noise vector $\vec{e}$ of $R$ using $R$ 's random tape, from which he can compute $R^{\prime}$ 's output $\mathrm{k}_{0}=(\vec{u}, \vec{v})$. For $i=1$ to $n$, Sim computes $m_{i, s_{i}}^{\prime}$ as $m_{i, s_{i}}+v_{i}$, and picks $m_{i, j}^{\prime} \stackrel{\$}{\leftarrow}\{0,1\}^{\lambda}$ for each $j \neq s_{i}$. Sim sends $\left(m_{i, j}^{\prime}\right)_{i, j}$ to $R$. By the security of $\Pi_{\mathrm{sVOLE}-G e n}$ and $G_{\mathrm{OT}}$, the $m_{i, j}^{\prime}$ for $j \neq s_{i}$ are indistinguishable from random from the viewpoint of $R$, hence the simulation is indistinguishable from an honest run of the protocol.

5.3.2 NISC for OT with Silent Preprocessing. Our 2-round OT extension protocol does actually directly give rise to a non-interactive secure computation protocol for the oblivious transfer functionality, with silent preprocessing, as defined in Section 3.2. For the sake of concreteness, we frame our OT extension protocol into the language of NISC with silent preprocessing on Figure 11.

5.3.3 Semi-Honest NISC for Reverse Subfield-VOLE. The same derandomization strategy as above directly implies, starting from the non-interactive PCG protocol for subfield-VOLE of Section 5.2, a NISC protocol for reverse subfield-VOLE with silent preprocessing, with features comparable to that of the NISC for OT extension. We omit the details.

\section{Protocol $\Pi_{\mathrm{OT}}$}

Parameters: $1^{\lambda}, n, N=2^{\ell}, t, p, r \in \mathbb{N} . H \in \mathbb{F}_{p}^{N \times n}$. PPRF is a puncturable PRF with domain $[N]$ and range $\mathbb{F}_{p^{r}} \cdot \mathcal{D}_{t, N}$ is a weight- $t$ error distribution over $\mathbb{F}_{p}^{N}$.

INPUTS:

- R inputs $n$ field elements $\left(s_{i}\right)_{i \leq n} \in \mathbb{F}_{p}^{n}$.

- $\mathrm{S}$ inputs $n$ length- $p$ vectors $\left(\vec{m}_{i}\right)_{i \leq n}$ where each $\vec{m}_{i}$ is over $\left(\{0,1\}^{\lambda}\right)^{p}$.

PROTOCOL:

(1) R picks $\vec{e} \stackrel{\$}{\leftarrow} \mathcal{D}_{t, N}$. Let $\mathcal{S}=\left\{\alpha_{1}, \ldots, \alpha_{t}\right\} \in[N]^{t}$ be the sorted indices of non-zero entries in $\vec{e}$, and $y_{i}=e_{\alpha_{i}} \in \mathbb{F}_{p}^{*}$. $\mathrm{R}$ computes the first part $\vec{u}$ of $G_{\mathrm{OT}}$. Expand $\left(0, \mathrm{k}_{0}\right)$ (note that $\vec{u}$ is computed as $\vec{e} \cdot H$ where $\vec{e}$ depends solely on $\vec{e}$ ). $\mathrm{R}$ sets $\vec{t} \leftarrow \vec{u}+\vec{s}$

(2) S samples $x \stackrel{\$}{\leftarrow} \mathbb{F}_{p^{r}}$ and $\mathrm{k}_{\text {pprf }} \stackrel{\$}{\leftarrow} \operatorname{PPRF} . \operatorname{Gen}\left(1^{\lambda}\right)$. He sets $\mathrm{k}_{1} \leftarrow\left(\mathrm{k}_{\text {pprf }}, x\right)$ and computes $\left\{w_{i, j}\right\}_{i \leq n, j \leq p} \leftarrow$ $G_{\text {OT }}$. Expand $\left(1, \mathrm{k}_{1}\right)$.

(3) R computes and sends to $S$ the first round of $\Pi_{s}$ VOLE-Gen on input $\vec{e}$, together with $\vec{t}$.

(4) $\mathrm{S}$ computes and sends to $\mathrm{R}$ the second round of $\Pi_{\text {sVOLE-Gen }}$ on input $\left(x, \mathrm{k}_{\text {pprf }}\right)$ together with $m_{i, j}^{\prime} \leftarrow$ $m_{i, j}+w_{i, t_{i}-j}$ for $i=1$ to $n$ and $j=1$ to $p$; R gets an output $\mathrm{k}_{0}$.

(5) R computes $(\vec{u}, \vec{v}) \leftarrow G_{\mathrm{OT}}$. Expand $\left(0, \mathrm{k}_{0}\right)$ and outputs $\left(m_{i, s_{i}}^{\prime}-v_{i}\right)_{i \leq n}$

Figure 10: Two-Round OT Extension

Theorem 5.5. Suppose the $\left(\mathcal{H} \mathcal{W}_{t}, H, \mathbb{F}_{p}\right)$-dual-LPN $\left(n^{\prime}, n\right)$ assumption holds. Then there is a semi-honest NISC protocol for reverse subfield-VOLE with silent preprocessing for generating length-n reverse subfield-VOLEs over an arbitrary field $\mathbb{F}_{p}$, which uses o(n) black-box executions of a 2-message semi-honest 1-out-of-2 $O T$, $O(n)$ black-box calls to a length-doubling PRG, one black-box call to a 2-message semi-honest reverse VOLE, and additionally computes one matrix-vector multiplication with $H$. It has total communication $(2 s+1) \cdot n+o(n)$.

\section{MALICIOUS DISTRIBUTED SETUP}

In this section, we present protocols for VOLE, OT extension and NISC with security against malicious parties. Our final protocol for OT extension takes place in four rounds, and can be compressed to two rounds via Fiat-Shamir.

We begin in Section 6.1 by formalizing and describing an augmented PPRF primitive with a "malicious key verification" procedure, corresponding to the event of when a selective-failure attack will (or not) be identified. The described selective-failure-only security notion is formalized and achieved for distributed generation of a single PPRF key in Section 6.2, and for $t$ PPRF keys (with consistent $x$ ) in Section 6.3. Then, in Section 6.4, we build atop this functionality to obtain a PCG protocol for subfield VOLE with standard malicious security. In the full version we explain how the PCG protocol for subfield VOLE can be converted into a four round PCG 


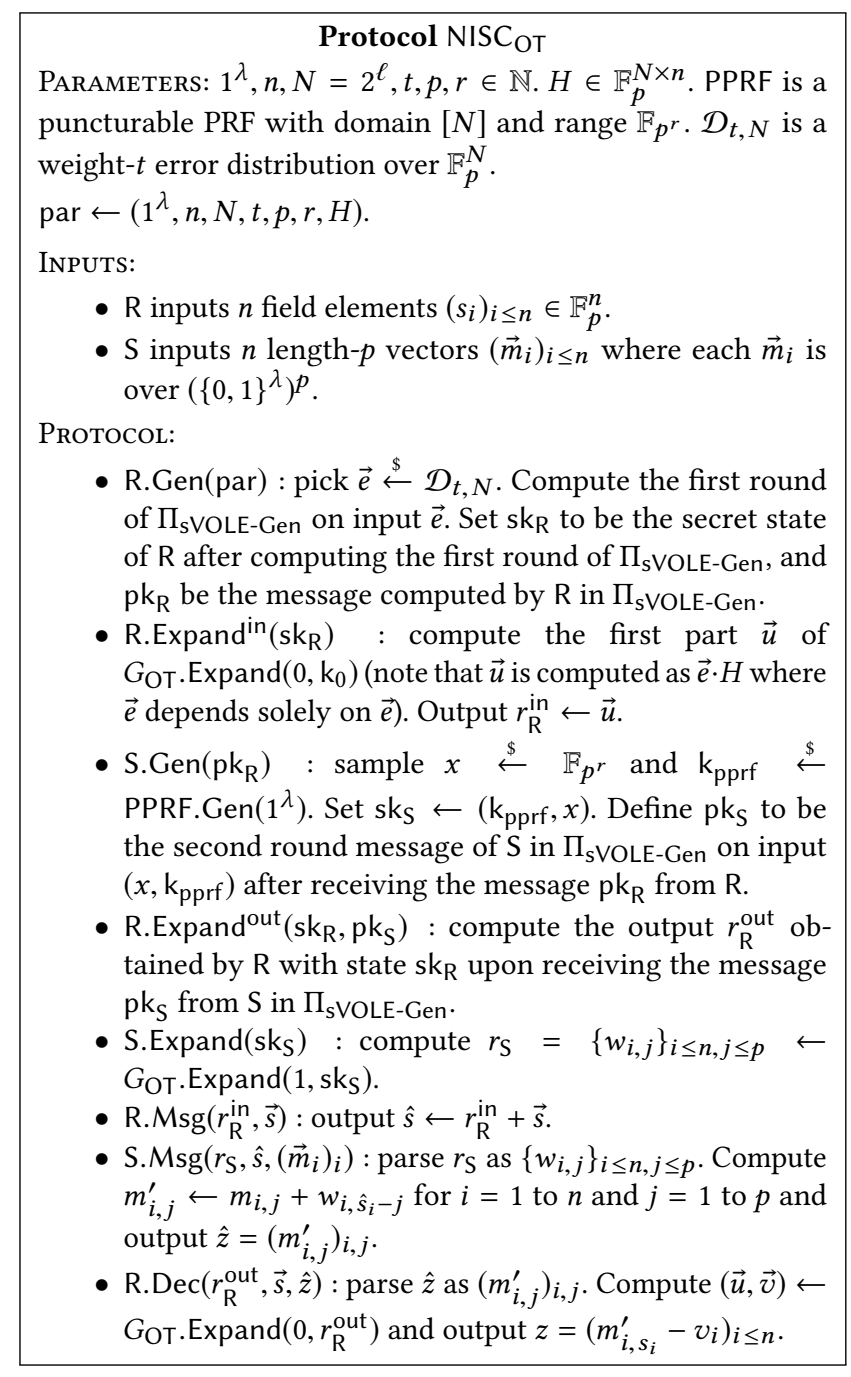

Figure 11: Non-interactive secure computation with silent preprocessing for oblivious transfer

protocol for random 1-out-of-p OT correlation. Finally, in the full version we also show how to apply the Fiat-Shamir heuristic to compress the protocol down to just 2 rounds, relying on a slightly stronger assumption. To obtain silent NISC for the OT functionality, which implies two-round OT extension on chosen inputs, we use the observation (as in our semi-honest protocol) that the receiver and sender can derandomize their inputs in parallel with their protocol messages. For details and the complete protocol for two-round malicious 1-out-of-2 OT extension, we refer to the full version.

\subsection{Puncturable PRF with Malicious Keys}

In the following sections we will realize a relaxed form of distributed PPRF setup functionality, where a corrupt sender may choose its own "master key," defining a PRF evaluation that need not coincide with any honest GGM tree, provided that it is consistent with the receiver's punctured point. The consistency check will serve as the "getting caught" predicate in our ideal functionality. In this section, we introduce necessary terminology in order for the consistency check to be formulated.

Roughly, we will have a malicious key space $\mathcal{K}$, such that given a malicious key $K \in \mathcal{K}$ and a subset of values $I \subseteq \mathcal{X}$ in the domain, one can check whether puncturing $K$ at any of the points in the set $I$ yields a consistent output. For a formal definition and instantiation for the GGM puncturable PRF, we refer to the full version.

In order to allow for a consistency check we use the GGM construction with domain $[2 N]$ and range $\left(\mathbb{F}_{p^{r}}\right)^{2} \times\{0,1\}^{\lambda}$ (where the former is the range of the left leaves and the latter the range of the right leaves). We will use an output $((\omega, w), \gamma) \in\left(\mathbb{F}_{p}\right)^{2} \times\{0,1\}^{\lambda}$ as follows: The value $w$ will correspond to the actual output of the PPRF. The value $\gamma$ will be used to ensure consistency within a single $P P R F$ evaluation. The value $\omega$ will be used to ensure consistency across $t$ PPRF evaluations.

\subsection{Malicious Setup for Single-Point PPRF}

As mentioned in the previous section, in order to achieve malicious security of a single PPRF evaluation, we use the redundancy introduced via the domain extension for checking consistent behaviour, by letting the sender provide a hash of all right leaves of the fully evaluated GGM tree. The idea is that a sender computing the correct hash value (relative to the receivers input $\alpha$ ), either behaved honestly, or guessed a set $I$ such that $\alpha \in I$. This is captured in the functionality in Figure 12. The functionality is similar to the semi-honest functionality given in Figure 12, but the adversary is additionally allowed to give a set $I \subseteq[N]$ as guess. If indeed $\alpha \in I$, the sender will successfully finish the protocol and learn some partial information about $\alpha$ (namely, $\alpha \in I$ ). Otherwise, the functionality will abort.

In order for the right leaves of the GGM tree to fix a unique tree, we require the PRG of the last level $G^{\prime}:\{0,1\}^{\lambda} \rightarrow\left(\mathbb{F}_{p^{r}}\right)^{2} \times\{0,1\}^{\lambda}$ to satisfy an additional property we call right-half injectivity, formally defined in the full version.

The protocol we present implements the functionality for the $\mathrm{PPRF}_{1}$, which corresponds to the GGM PPRF, but where evaluation returns a value in $\left(\mathbb{F}_{p^{r}}\right)^{2}$ instead of $\left(\mathbb{F}_{p^{r}}\right)^{2} \times\{0,1\}^{\lambda}$.

We give the protocol for distributed setup of $\mathrm{PPRF}_{1}$ with security against malicious adversaries in Figure 13. First, in steps 1-6 the parties run the semi-honest protocol, such that the receiver holds a key $\mathrm{k}^{*}$ punctured at $\alpha \| 0$ and the sender a possibly malicious key $K$. As the tree is always punctured at an even value, both parties can compute all the right leaves of the GGM tree. The sender additionally sends a hash of all these leaves to the receiver. The receiver checks if this hash is consistent with his view and aborts otherwise.

THEOREM 6.1. Assuming a black-box access to a PRGG $:\{0,1\}^{\lambda} \mapsto$ $\{0,1\}^{2 \lambda}$, a right-half injective $P R G G^{\prime}:\{0,1\}^{\lambda} \mapsto\left(\mathbb{F}_{p^{r}}\right)^{2} \times\{0,1\}^{\lambda}$, and a collision resistant hash function $h:\{0,1\}^{\lambda N} \rightarrow\{0,1\}^{\lambda}$, there exists a 2-party protocol implementing $\mathcal{F}_{\text {mal-PPRF }}$ (see Fig. 12) for the puncturable PRF $\mathrm{PPRF}_{1}$, with malicious security in the parallel OThybrid model, and the following efficiency features. The interaction consists of $\ell$ parallel calls to $\mathcal{F}_{\mathrm{OT}}$, and uses additional communication of $r \log p+\lambda$. The computational complexity is dominated by $O\left(2^{\ell}\right)$ calls each to $G$ and $G^{\prime}$. 


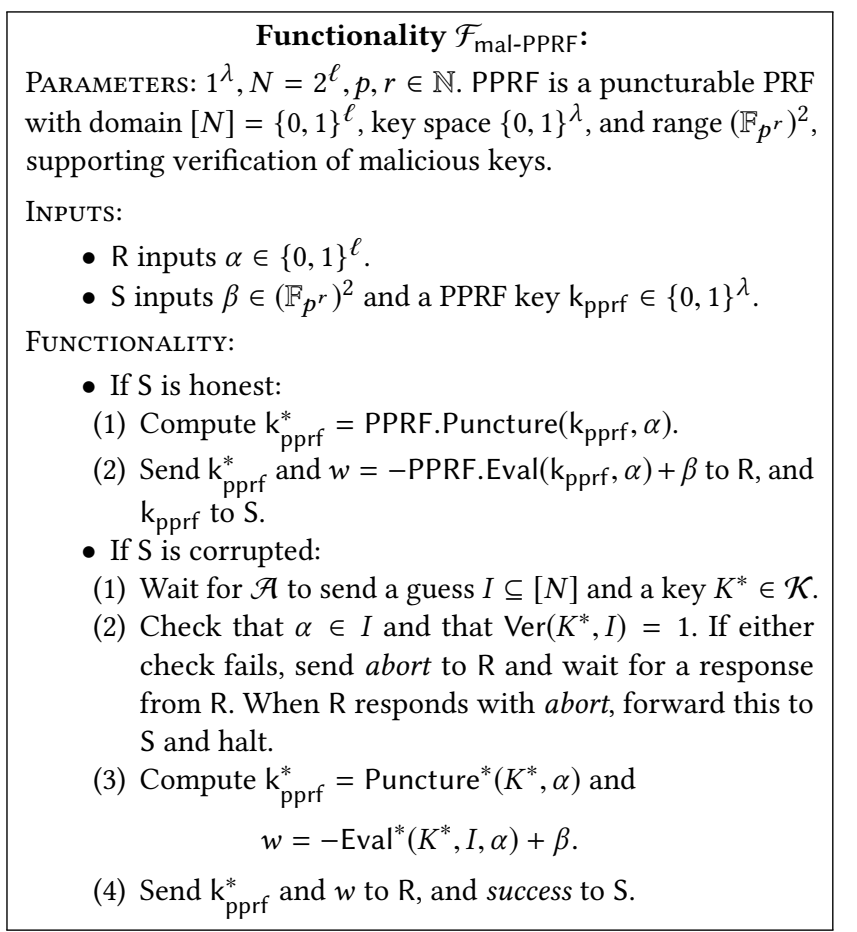

Figure 12: Functionality for malicious distributed setup of single-point PPRF

\subsection{Malicious Setup of $t$ PPRFs with Consistent Offset}

For the VOLE setup with malicious security, we require a protocol for distributed setup of $t$ PPRFs, where the inputs $\beta_{j}$ of the sender are consistent across all evaluations. By consistent, we mean that each $\beta_{j}$ is an additive share of $x \cdot y_{j}$, where the receiver knows the other share and the noise value $y_{j} \in \mathbb{F}_{p}^{*}$. To this end, we introduce a second consistency check, where the sender has to provide a linear combination of the outputs of each PPRF. We show that a cheating sender will fail this final check, unless he managed to guess part of the receiver's input. This guessing is modelled by the functionality $\mathcal{F}_{\text {mal- } t \text {-PPRF }}$ (Fig. 15), which is parameterized by a 1-puncturable PRF with verification of malicious keys.

To carry out this check, we exploit the extended range of the PPRF given by the functionality $\mathcal{F}_{\text {mal-PPRF. The extra }} \mathbb{F}_{p^{r}}$ element from each evaluation serves to check consistency, by taking a random linear combination of all these outputs (for each PPRF), together with a linear combination of the original outputs, and sending these to the receiver to check. Note that without the extended range, sending a linear combination of PPRF outputs to the receiver would leak the sender's input $x$; with the extra outputs, however, the sender can use a random value $\chi$ which serves to mask $x$.

Since we sacrifice the extended outputs in the consistency check, the functionality $\mathcal{F}_{\text {mal- } t \text {-PPRF which we realize gives us a PPRF with }}$ range $\mathbb{F}_{p^{r}}$, which is defined by simply ignoring the first element output from the one with range $\mathbb{F}_{p^{r}}^{2}$.
Protocol $\Pi_{\text {mal-PPRF: }}$
PARAmeters: $1^{\lambda}, \ell, N=2^{\ell}, p, r \in \mathbb{N}$. PPRF GGM $_{\text {is }}$ ise GGM puncturable PRF with domain $\{0,1\}^{\ell+1}=[2 N]$, key space $\{0,1\}^{\lambda}$, and range $\left(\mathbb{F}_{p^{r}}\right)^{2} \times\{0,1\}^{\lambda}$, constructed from a lengthdoubling PRG $G:\{0,1\}^{\lambda} \mapsto\{0,1\}^{2 \lambda}$, and a second PRG $G^{\prime}:\{0,1\}^{\lambda} \mapsto\left(\mathbb{F}_{p^{r}}\right)^{2} \times\{0,1\}^{\lambda}$ used to compute the PRF outputs on the last level of the tree.

INPUTS:

- R inputs $\alpha \in\{0,1\}^{\ell}$.

- S inputs $\beta \in\left(\mathbb{F}_{p^{r}}\right)^{2}$ and a PPRF key kprf $\in\{0,1\}^{\lambda}$.

PRotocol:

(1) $\mathrm{S}$ samples a random seed $\mathrm{k}_{\mathrm{pprf}} \in\{0,1\}^{\lambda}$.

(2) $\mathrm{S}$ computes the $2^{i}$ partial evaluations at level $i$ of the GGM tree:

(a) S sets $s_{0}^{0}=\mathrm{k}_{\text {pprf }}$.

(b) For $i \in\{1, \ldots, \ell\}, j \in\left[0, \ldots, 2^{i-1}\right)$ : $\mathrm{S}$ computes $\left(s_{2 j}^{i}, s_{2 j+1}^{i}\right)=G\left(s_{j}^{i-1}\right)$.

(c) For $j \in\{0,1\}^{\ell}: \mathrm{S}$ computes $\left(s_{2 j}^{\ell+1}, s_{2 j+1}^{\ell+1}\right)=G^{\prime}\left(s_{j}^{\ell}\right) \in$ $\left(\mathbb{F}_{p^{r}}\right)^{2} \times\{0,1\}^{\ell}$.

(3) S computes the "left" and "right" halves for $i \in\{1, \ldots, \ell\}$ :

$$
K_{0}^{i}=\bigoplus_{j \in\left[0,2^{i-1}\right)} s_{2 j}^{i}, \quad K_{1}^{i}=\bigoplus_{j \in\left[0,2^{i-1}\right)} s_{2 j+1}^{i}
$$

(4) S computes the "right" half for $i=\ell+1$ :

$$
K_{1}^{\ell+1}=\bigoplus_{j \in\{0,1\}^{\ell}} s_{2 j+1}^{\ell+1}
$$

(5) For $i=1, \ldots, \ell=\log N$ (in parallel) the parties run OT where in the $i$-th OT:

(a) $\mathrm{R}$ inputs the choice bit $\overline{\alpha_{i}}$.

(b) $\mathrm{S}$ inputs the pair $\left(K_{0}^{i}, K_{1}^{i}\right)$.

(6) $\mathrm{S}$ sends to $\mathrm{R}$ the key $K_{1}^{\ell+1}$ and the correction value

$$
c=\beta-\sum_{j \in[N]} s_{2 j}^{\ell+1} .
$$

(7) For the consistency check, S sets $\gamma_{j}=s_{2 j+1}^{\ell+1}$ for all $j \in[N]$ and sends to $\mathrm{R}$ the value $\Gamma=h\left(\gamma_{0}, \ldots, \gamma_{N-1}\right)$.

(8) Let $\left\{K^{i}\right\}_{i=1}^{\ell+1}$ denote the OT outputs received by $\mathrm{R}$ together with the key of the $(\ell+1)$-st level. Then, $\mathrm{R}$ proceeds as follows.

(a) $\mathrm{k}_{\text {pprf }}^{*} \leftarrow$ Puncture $^{*}\left(\left\{K^{i}\right\}_{i=1}^{\ell+1}, \alpha\right)$.

(b) $\left\{s_{j}\right\}_{j \neq \alpha \| 0} \leftarrow \operatorname{PPRF}_{\mathrm{GGM}}$. FullEval $\left(\mathrm{k}_{\mathrm{pprf}}^{*}, \alpha \| 0\right)$.

(c) $\mathrm{R}$ receives $c$, and computes

$$
w=c-\sum_{j \in[N] \backslash\{\alpha\}} s_{2 j}
$$

(d) To verify consistency, R sets $\gamma_{j}=s_{2 j+1}$ for all $j \in[N]$, and computes $\Gamma^{\prime}=h\left(\gamma_{0}, \ldots, \gamma_{N-1}\right)$.

(9) If $\Gamma=\Gamma^{\prime}$, R outputs the punctured key $k_{\mathrm{pprf}}^{*}$, and the final correction value $w$. Otherwise, R aborts.

Figure 13: Protocol for distributed setup of single-point PPRF with consistency check 


Functionality $\mathcal{F}_{\text {g-rev-VOLE }}$
PARAmETERs: $t, p, r \in \mathbb{N}$.
InPUT: The sender S inputs a pair $((\vec{\beta}, \chi),(\vec{b}, x)) \in\left(\mathbb{F}_{p^{r}}^{t} \times \mathbb{F}_{p^{r}}\right)^{2}$.
The receiver R inputs a vector $\vec{y} \in \mathbb{F}_{p}^{t}$.
Functionality: Compute $\vec{\gamma} \leftarrow \vec{y} \chi-\vec{\beta}$ and $\vec{c} \leftarrow \vec{y} x-\vec{b}$ and
output $(\vec{\gamma}, \vec{c})$ to R.

Figure 14: Generalized Reverse Vector-OLE Functionality over a Field $\mathbb{F}_{p}$

\section{Functionality $\mathcal{F}_{\text {mal- } t \text {-PPRF }}$}

Parameters: $1^{\lambda}, N=2^{\ell}, t, p, r \in \mathbb{N}$. PPRF is a puncturable PRF with domain $[N]$, key space $\{0,1\}^{\lambda}$, and range $\mathbb{F}_{p^{r}}$, supporting verification of malicious keys.

INPUTS:

- $\mathrm{R}$ inputs indices $\alpha_{1}, \ldots, \alpha_{t} \in[N]$ and weights $y_{1}, \ldots, y_{t} \in \mathbb{F}_{p}^{*}$. We define $\mathcal{S}=\left\{\alpha_{1}, \ldots, \alpha_{t}\right\}$.

- $\mathrm{S}$ inputs $\mathrm{k}_{1}=\left(\left\{\mathrm{k}_{j}\right\}_{j \in[t]}, x\right)$, where $x \in \mathbb{F}_{p^{r}}$ and $\mathrm{k}_{j} \in$ $\{0,1\}^{\lambda}$.

FunCtionality:

- If $\mathrm{S}$ is honest:

(1) Compute $\mathrm{k}_{j}^{*} \leftarrow$ PPRF.Puncture $\left(\mathrm{k}_{j}, \alpha_{j}\right)$, for $j \in$ $\{1, \ldots, t\}$.

(2) Let $z_{j} \leftarrow x \cdot y_{j}-\operatorname{PPRF} . \operatorname{Eval}\left(\mathrm{k}_{j}, \alpha_{j}\right)$ for $j \in\{1, \ldots, t\}$.

(3) Let $\mathrm{k}_{0} \leftarrow\left(\left\{\mathrm{k}_{j}^{*}, z_{j}\right\}_{j \in[t]}, \mathcal{S}, \vec{y}\right)$.

(4) Output $k_{0}$ to R.

- If $\mathrm{S}$ is corrupted:

(1) Receive from $\mathcal{A} t$ subsets $I_{1}, \ldots, I_{t} \subseteq[N]$ and a set of keys $K_{1}^{*}, \ldots, K_{t}^{*} \in \mathcal{K}$.

(2) For each $j \in\{1, \ldots, t\}$ check that $\alpha_{j} \in I_{j}$ and that $\operatorname{Ver}\left(K_{j}^{*}, I_{j}\right)=1$. If any check fails, abort.

(3) Compute $k_{j}^{*}=$ PPRF.Puncture ${ }^{*}\left(K_{j}^{*}, \alpha_{j}\right)$ for each $j \in$ $\{1, \ldots, t\}$.

(4) Let $z_{j} \leftarrow x \cdot y_{j}-\operatorname{PPRF} . \mathrm{Eval}^{*}\left(K_{j}^{*}, I_{j}, \alpha_{j}\right)$ for $j \in$ $\{1, \ldots, t\}$.

(5) Output $\mathrm{k}_{0} \leftarrow\left(\mathrm{k}_{\mathrm{pprf}}^{*}, \mathcal{S}, \vec{y},\left\{z_{j}\right\}_{j \in[t]}\right)$ to $\mathrm{R}$ and success to $\mathrm{S}$.

Figure 15: Functionality for malicious distributed setup of $t$ puncturable PRFs

To create the shares of $x \cdot y_{j}$, when $p>2$ we again need a slightly stronger flavor of reverse VOLE, presented in Figure 14. Here, we require the functionality to take two inputs by the sender $((\vec{\beta}, \chi),(\vec{b}, x))$, and only one input $\vec{y}$ by the receiver, and return to the sender values $\vec{\gamma}, \vec{c}$, such that $(\vec{\beta}, \vec{\gamma})$ constitute sharings of $x \times \vec{y}$ (and similar for $\vec{c}$ ). Note that it is not enough for our protocol to instead call the basic reverse VOLE functionality twice, as a receiver providing inconsistent inputs in the two calls can learn the input $x$ of the sender in the protocol $\Pi_{\text {mal- } t \text {-PPRF (Figure 16). }}$

For a proof of the following theorem we refer to the full version.

\section{Protocol $\Pi_{\text {mal- } t \text {-PPRF: }}$}

PARAmeters: $1^{\lambda}, N=2^{\ell}, t, p, r \in \mathbb{N}$. PPRF is a puncturable PRF with domain $[N]$, key space $\{0,1\}^{\lambda}$ and range $\left(\mathbb{F}_{p^{r}}\right)^{2}$.

INPUTS:

- R inputs distinct indices $\alpha_{1}, \ldots, \alpha_{t} \in[N]$ and weights $y_{1}, \ldots, y_{t} \in \mathbb{F}_{p}^{*}$. We define $\mathcal{S}=\left\{\alpha_{1}, \ldots, \alpha_{t}\right\}$ and $\vec{y}=$ $\left(y_{1}, \ldots, y_{t}\right) \in\left(\mathbb{F}_{p}^{*}\right)^{t}$.

- S inputs $x \in \mathbb{F}_{p^{r}}$.

PRotocol:

(1) S picks $\vec{\beta}, \vec{b} \stackrel{\$}{\leftarrow} \mathbb{F}_{p^{r}}^{t}$ and $\chi \stackrel{\$}{\leftarrow} \mathbb{F}_{p^{r}}$.

(2) $\mathrm{R}$ and $\mathrm{S}$ call $\mathcal{F}_{\text {g-rev-VOLE }}(t, p, r)$ on respective inputs $\vec{y}$ and $((\vec{\beta}, \chi),(\vec{b}, x))$. R receives $(\vec{\gamma}, \vec{c}) \in \mathbb{F}_{p^{r}}^{t} \times \mathbb{F}_{p^{r}}^{t}$.

(3) $\mathrm{R}$ and $\mathrm{S}$ call $\mathcal{F}_{\text {mal-PPRF}}\left(1^{\lambda}, N, p, r\right) t$ times on respective inputs $\alpha_{j}$ and $\left(\beta_{j}, b_{j}\right)$. R receives $\mathrm{k}_{j}^{*}$ and $\left(\omega_{j}, w_{j}\right)$ for each $j \in\{1, \ldots, t\}$. If any of the runs is not successful, $\mathrm{R}$ receives abort from the functionality.

(4) R samples $\tau, \tau_{0}, \ldots, \tau_{N-1} \stackrel{\$}{\leftarrow} \mathbb{F}_{p^{r}}$ and sends these to $\mathrm{S}$.

(5) $\mathrm{S}$ computes $\left(v_{S, 2 i}^{j}, v_{S, 2 i+1}^{j}\right) \leftarrow \operatorname{PPRF} . \operatorname{Eval}\left(\mathrm{k}_{j}, i\right)$ for $i \in$ $[N], j \in\{1, \ldots, t\}$, and sends $X=\chi+\tau \cdot x$ and $V_{S, j}=$ $\sum_{i=0}^{N-1} \tau_{i} \cdot\left(v_{S, 2 i}^{j}+\tau \cdot v_{S, 2 i+1}^{j}\right)$ for $j \in\{1, \ldots, t\}$ to $\mathrm{R}$.

(6) R computes $\left(v_{R, 2 i}^{j}, v_{R, 2 i+1}^{j}\right) \leftarrow \operatorname{PPRF} . \operatorname{Eval}^{\prime}\left(\mathrm{k}_{j}^{*}, i\right)$ for $i \in[N], j \in\{1, \ldots, t\}$, where PPRF.Eval' is an algorithm that outputs $\left(\omega_{j}, w_{j}\right)+\left(\gamma_{j}, c_{j}\right)$ on input $\left(\mathrm{k}_{j}^{*}, \alpha_{j}\right)$, and -PPRF.Eval $\left(\mathrm{k}_{j}^{*}, i\right)$ else.

(7) R checks if $V_{S, j}+\sum_{i=0}^{N-1} \tau_{i} \cdot\left(v_{R, 2 i}^{j}+\tau \cdot v_{R, 2 i+1}^{j}\right)=X \cdot \tau_{\alpha_{j}} \cdot y_{j}$ for $j \in\{1, \ldots, t\}$. If any of these checks fail or $\mathrm{R}$ received abort from $\mathcal{F}_{\text {mal-PPRF }}$ in step $3, \mathrm{R}$ aborts. Otherwise, $\mathrm{R}$ sends $o k$ to $\mathcal{F}_{\text {mal-PPRF }}$ and outputs $\mathrm{k}_{0}=\left(\left\{\mathrm{k}_{j}^{*}, z_{j}\right\}_{j}, \mathcal{S}, \vec{y}\right)$.

Figure 16: Protocol for malicious distributed setup of $t$ puncturable PRFs

THEOREM 6.2. There exists a 4-message 2-party protocol $\Pi_{\text {mal- } t \text {-PPRF }}$ which securely implements the functionality $\mathcal{F}_{\text {mal- } t \text {-PPRF }}\left(1^{\lambda}, N, p, r\right)$ for the puncturable PRFPPRF in the $\mathcal{F}_{\text {g-rev-VOLE }}{ }^{-}$, parallel $\mathcal{F}_{\text {mal-PPRF }}{ }^{-}$ hybrid model, with malicious security, using $t$ parallel calls to $\mathcal{F}_{\text {mal-PPRF, }}$ and only one call to $\mathcal{F}_{\text {g-rev-VOLE, }}$ and further communication of $(N+$ $t+2) r \log p$ bits. Furthermore, when $p=2$, the functionality can be implemented in the parallel $\mathcal{F}_{\text {mal-PPRF}}$-hybrid model, using no call to $\mathcal{F}_{\text {g-rev-VOLE. }}$

Note that an additional PRG with range $\mathbb{F}_{p^{r}}^{N+1}$, the communication can be reduced to just $(t+1) r \log p+\lambda$ bits.

\subsection{4-Round VOLE and OT Setup with Malicious Security}

The $\mathcal{F}_{\text {mal- } t \text {-PPRF }}$ functionality can be immediately used to distribute the setup of the subfield-VOLE PCG from Section 4. To prove this gives secure subfield-VOLE, however, we now need to assume that the dual-LPN assumption remains secure when an adversary is allowed to query (on average) one bit of information on the error 
vector. This reflects the fact that a malicious sender in $\mathcal{F}_{\text {mal-t-PPRF }}$ can try to guess subsets containing the receiver's $\alpha_{j}$ inputs, which correspond to non-zero coordinates of the error vector. This assumption with leakage is essentially the same as an assumption recently used for maliciously secure MPC based on syndrome decoding [34]. For a formal definition we refer to the full version.

Theorem 6.3. Suppose that $\left(\mathcal{H} \mathcal{W}_{t}, \mathrm{C}, \mathbb{F}_{p}\right)$-dual-LPN $(N, n)$ with static leakage holds, where $N=O(n)$ and $t=o(n /(\lambda \log n)$. Then there exists a 4-message, maliciously secure PCG protocol for the subfield VOLE correlation, which makes o( $n$ ) parallel calls to an oblivious transfer functionality, with communication complexity o(n) bits.

\section{IMPLEMENTATION}

\subsection{Instantiating the Code and Parameters}

The most costly part of our implementation is the syndrome computation with the matrix $H$ used in the dual-LPN assumption. We optimize this by instantiating $H$ using the parity-check matrix of a quasi-cyclic code. Multiplication by $H$ can then be done with polynomial arithmetic in $\mathbb{Z}_{2}[X] /\left(X^{n}-1\right)$, for which we use the library bitpolymul [21]. Another optimization that improves efficiency and reduces the seed size is to use a regular error distribution, where the error vector $\vec{e} \in \mathbb{F}_{2}^{N}$ is the concatenation of $t$ random unit vectors, each of length $N / t$. To choose the code parameters $N, n$ and the error weight $t$, we analyze security against the best known attacks, additionally accounting for a $\sqrt{N}$ speedup that can be obtained from the DOOM attack [53] when using quasi-cyclic codes. As also observed in [35], we are not aware of any attacks that exploit regular errors and perform significantly better than usual.

In the full version, we provide more details on selecting parameters and describe some further optimizations for the syndrome computation.

\subsection{Results}

We implement our semi-honest and malicious secure protocols and report their performance in several different settings. The source code can be found at https://github.com/osu-crypto/libOTe. The benchmark was performed on a single AWS c4.4xLarge instance with network latency artificially limited to emulate a LAN or WAN settings. Specifically, we consider a LAN setting with bandwidth of $10 \mathrm{Gbps}$ and $0 \mathrm{~ms}$ latency and two WAN settings with 100, 10 Mbps \& 40ms one-way latency. We compare with the semi-honest OT extension protocol of Ishai et al. [37] (IKNP) and the malicious secure protocol of [41] (KOS) as implemented by a state-of-theart library. Both our implementations and that of [37, 41] use the same three round malicious secure base OT protocol of Naor \& Pinkas[47]. We note that our protocols can be composed with a two round base OT protocol to give a two round OT extension. In the WAN setting this optimization would reduce the running times by approximately $40 \mathrm{~ms}$ for all protocols.

The functionality we realize is to produce $n \in\left\{10^{4}, 10^{5}, 10^{6}, 10^{7}\right\}$ uniformly random OTs of length 128 bits. One distinction between our protocol and $[37,41]$ is that the choice bits of the receiver are uniformly chosen by our protocol, while $[37,41]$ allows the receiver to specify them. These random OTs can then be de-randomized with additional communication.

Figure 17 contains the running time of our protocol. A fuller table, with alternative choices of parameters (security parameter $\lambda$, scaling parameter $N / n$, method for computing the base OTs) is available in the full version. The primary takeaway is that both of our protocols achieve extremely low communication while the total running time remains competitive with or superior to KOS and IKNP. We report running times with each party having 1 or 4 threads, along with a background IO thread. In the LAN setting with sub-millisecond latency \& 10Gbps we observe that the IKNP and KOS protocols achieve significant performance, requiring just 0.26 or 0.33 seconds to compute 10 million OTs with a single thread. While the computational cost of IKNP and KOS does outperform our implementation by roughly one order of magnitude, it also requires between 1000 and 2000 times more communication. This difference means that for more realistic network settings, such as $100 \mathrm{Mbps}$, our implementation achieves a faster running time. With 4 threads and a limit of $100 \mathrm{Mbps}$ our implementation is up to 5 times faster (counting total running time, including both local computation and communication costs) and remains faster even for small $n$ where our communication overheads are asymptotically closer together.

For the constrained setting of $10 \mathrm{Mbps}$ our protocol truly stands out with a 47 times speedup compared to IKNP with $n=10^{7}$ and $t=4$. We see a similar 46 times speedup in the malicious setting compared to KOS. Moreover, when comparing between the across the different network settings our protocol incurs minimal to no perform impact from decreasing bandwidth. For instance, with a 10Gbps connection our semi-honest protocol processes $n=10^{7}$ OTs in 2.4 seconds while with 1000 times less bandwidth the protocol still just requires 2.8 seconds.

This scalability is explained in Figure 18 which contains the communication overhead of our protocol. A fuller table, with alternative choices of parameters (security parameter $\lambda$, scaling parameter $N / n$, method for computing the base OTs) is available in the full version. We parameterize our protocols by the desired security level $\lambda \in\{80,128\}$ and a tunable parameter $s=N / n$. The latter controls a trade-off between the number of PPRF evaluations and length of the resulting vectors. To maintain security level of $\lambda$ bits, increasing $s$ results in fewer PPRF evaluations and less communication. However, it also increases the computational overhead. Our smallest running times were achieved with $s=2$. However, we also consider $s=4$ which decreases our total communication from $126 \mathrm{~KB}$ to $80 \mathrm{~KB}$ for $n=10^{7}$. In contrast, the IKNP protocol requires $160 \mathrm{MB}$ for the same security level. This represents as much as a 2000 times reduction in communication. This low communication overhead results in our protocol requiring as little as 0.038 bits per OT for $n=10^{7}$ and $\lambda=80$. In our worst case of $n=10^{4}$ our protocol still requires between 3 and 6 times less communication than IKNP. Another compelling property of our protocol is that we incur near constant additive communication overhead when comparing our malicious and semi-honest protocols.

\section{ACKNOWLEDGEMENTS}

E. Boyle, N. Gilboa, and Y. Ishai supported by ERC Project NTSC (742754). E. Boyle additionally supported by ISF grant 1861/16 and 


\begin{tabular}{|c|c|c|c|c|c|c|c|c|c|c|c|c|c|c|c|}
\hline \multirow[b]{2}{*}{ Protocol } & \multirow[b]{2}{*}{ Base type } & \multirow[b]{2}{*}{$\lambda$} & \multirow[b]{2}{*}{$\tau$} & \multicolumn{4}{|c|}{$\begin{array}{c}\text { LAN (10Gbps) times } \\
n\end{array}$} & \multicolumn{4}{|c|}{$\begin{array}{l}\text { WAN (100Mbps) times } \\
n\end{array}$} & \multicolumn{4}{|c|}{$\begin{array}{c}\text { WAN (10Mbps) times } \\
n\end{array}$} \\
\hline & & & & $10^{7}$ & $10^{6}$ & $10^{5}$ & $10^{4}$ & $10^{7}$ & $10^{6}$ & $10^{5}$ & $10^{4}$ & $10^{7}$ & $10^{6}$ & $10^{5}$ & $10^{4}$ \\
\hline This (SH) & hybrid & 128 & 4 & 2,441 & 208 & 76 & 67 & 2,726 & 513 & 422 & 425 & 2,756 & 518 & 454 & 422 \\
\hline IKNP & base & 128 & 4 & 268 & 125 & 94 & 91 & 13,728 & 1,850 & 493 & 459 & 128,954 & 13,332 & 1,756 & 445 \\
\hline This (SH) & hybrid & 128 & 1 & 7,990 & 533 & 130 & 100 & 8,252 & 808 & 451 & 422 & 8,291 & 815 & 467 & 422 \\
\hline IKNP & base & 128 & 1 & 573 & 157 & 108 & 98 & 15,622 & 2,030 & 613 & 341 & 129,011 & 13,285 & 1,672 & 429 \\
\hline This (Mal) & hybrid & 128 & 4 & 2,659 & 280 & 84 & 78 & 2,872 & 479 & 457 & 424 & 2,846 & 515 & 438 & 422 \\
\hline KOS & base & 128 & 4 & 333 & 121 & 110 & 111 & 13,722 & 1,933 & 589 & 426 & 129,052 & 13,391 & 1,804 & 536 \\
\hline This (Mal) & hybrid & 128 & 1 & 8,765 & 584 & 141 & 104 & 9,055 & 828 & 460 & 423 & 8,929 & 831 & 467 & 433 \\
\hline KOS & base & 128 & 1 & 674 & 170 & 113 & 106 & 15,741 & 2088 & 702 & 433 & 129,771 & 13,389 & 1,772 & 518 \\
\hline
\end{tabular}

Figure 17: The running time in milliseconds of our implementation compared to [8] in both the LAN (0ms latency) and WAN (40ms one-way latency) settings, with security parameter $\lambda=128 . \lambda$ is the computational security parameter. We set the scaling $N / n$ to 2. $\tau$ denotes the number of threads. Hybrid refers to doing 128 base OTs followed by IKNP to derive the total required base OTs.

\begin{tabular}{|c|c|c|c|c|c|c|c|c|c|}
\hline \multirow[b]{3}{*}{ Protocol } & \multirow[b]{3}{*}{ Base type } & \multicolumn{4}{|c|}{ Total Comm. (bytes) } & \multicolumn{4}{|c|}{ Comm./OT (bits) } \\
\hline & & & $n$ & & & & ${ }^{r}$ & & \\
\hline & & $10^{7}$ & $10^{6}$ & $10^{5}$ & $10^{4}$ & $10^{7}$ & $10^{6}$ & $10^{5}$ & $10^{4}$ \\
\hline This (SH/Mal) & hybrid & 126,658 & 98,754 & 83,394 & 57,806 & 0.101 & 0.790 & 6.672 & 46.245 \\
\hline IKNP/KOS & base & $160,056,360$ & $16,011,518$ & $1,655,784$ & 168,186 & 128.045 & 128.092 & 132.463 & 134.549 \\
\hline
\end{tabular}

Figure 18: The communication overhead of our implementation compared to [37, 41], with $N / n=2$ and $\lambda=4$. See Figure 17 .

AFOSR Award FA9550-17-1-0069. G. Couteau supported by ERC Project PREP-CRYPTO (724307). N. Gilboa additionally supported by ISF grant $1638 / 15$, ERC grant 876110 , and a grant by the BGU Cyber Center. Y. Ishai additionally supported by ISF grant 1709/14, NSF-BSF grant 2015782, DARPA SPAWAR contract N66001-15-C4065, and a grant from the Ministry of Science and Technology, Israel and Department of Science and Technology, Government of India. L. Kohl supported by ERC Project PREP-CRYPTO (724307) and by DFG grant HO 4534/2-2. This work was done in part while visiting the FACT Center at IDC Herzliya, Israel. P. Scholl supported by the European Union's Horizon 2020 research and innovation programme under grant agreement No 731583 (SODA), and the Danish Independent Research Council under Grant-ID DFF-610800169 (FoCC).

\section{REFERENCES}

[1] Afshar, A., Mohassel, P., Pinkas, B., Riva, B.: Non-interactive secure computation based on cut-and-choose. In: Nguyen, P.Q., Oswald, E. (eds.) EUROCRYPT 2014 LNCS, vol. 8441, pp. 387-404. Springer, Heidelberg (May 2014)

[2] Aguilar Melchor, C., Aragon, N., Bettaieb, S., Bidoux, L., Blazy, O., Deneuville, J.C., Gaborit, P., Persichetti, E., Zémor, G.: Hamming quasi-cyclic (HQC) (2019), https://pqc-hqc.org/doc/hqc-specification_2018-12-14.pdf

[3] Alekhnovich, M.: More on average case vs approximation complexity. In: 44th FOCS. pp. 298-307. IEEE Computer Society Press (Oct 2003)

[4] Applebaum, B., Damgård, I., Ishai, Y., Nielsen, M., Zichron, L.: Secure arithmetic computation with constant computational overhead. In: Katz, J., Shacham, H (eds.) CRYPTO 2017, Part I. LNCS, vol. 10401, pp. 223-254. Springer, Heidelberg (Aug 2017)

[5] Applebaum, B., Ishai, Y., Kushilevitz, E.: Cryptography with constant input locality. Journal of Cryptology 22(4), 429-469 (Oct 2009)

[6] Aragon, N., Barreto, P., Bettaieb, S., Bidoux, L., Blazy, O., Deneuville, J.C., Gaborit, P., Gueron, S., Guneysu, T., Melchor, C.A., et al.: Bike: Bit flipping key encapsulation (2019), https://bikesuite.org/files/round2/spec/BIKE-Spec-2019.06.30.1.pdf

[7] Arora, S., Ge, R.: New algorithms for learning in presence of errors. In: Aceto, L., Henzinger, M., Sgall, J. (eds.) ICALP 2011, Part I. LNCS, vol. 6755, pp. 403-415.
Springer, Heidelberg (Jul 2011)

[8] Asharov, G., Lindell, Y., Schneider, T., Zohner, M.: More efficient oblivious transfer and extensions for faster secure computation. In: Sadeghi, A.R., Gligor, V.D., Yung, M. (eds.) ACM CCS 2013. pp. 535-548. ACM Press (Nov 2013)

[9] Beaver, D.: Efficient multiparty protocols using circuit randomization. In: Advances in Cryptology - CRYPTO '91, 11th Annual International Cryptology Conference, Santa Barbara, California, USA, August 11-15, 1991, Proceedings. pp. 420-432 (1991), https://doi.org/10.1007/3-540-46766-1_34

[10] Becker, A., Joux, A., May, A., Meurer, A.: Decoding random binary linear codes in $2^{n / 20}$ : How $1+1=0$ improves information set decoding. In: Pointcheval, D., Johansson, T. (eds.) EUROCRYPT 2012. LNCS, vol. 7237, pp. 520-536. Springer, Heidelberg (Apr 2012)

[11] Bendlin, R., Damgård, I., Orlandi, C., Zakarias, S.: Semi-homomorphic encryption and multiparty computation. In: Paterson, K.G. (ed.) EUROCRYPT 2011. LNCS, vol. 6632, pp. 169-188. Springer, Heidelberg (May 2011)

[12] Blum, A., Furst, M.L., Kearns, M.J., Lipton, R.J.: Cryptographic primitives based on hard learning problems. In: Advances in Cryptology - CRYPTO '93, 13th Annual International Cryptology Conference, Santa Barbara, California, USA, August 22-26, 1993, Proceedings. pp. 278-291 (1993), https://doi.org/10.1007/3540-48329-2 24

[13] Blum, A., Kalai, A., Wasserman, H.: Noise-tolerant learning, the parity problem, and the statistical query model. In: 32nd ACM STOC. pp. 435-440. ACM Press (May 2000)

[14] Boneh, D., Waters, B.: Constrained pseudorandom functions and their applications. In: Sako, K., Sarkar, P. (eds.) ASIACRYPT 2013, Part II. LNCS, vol. 8270, pp. 280-300. Springer, Heidelberg (Dec 2013)

[15] Boyle, E., Couteau, G., Gilboa, N., Ishai, Y.: Compressing vector OLE. In: Lie, D., Mannan, M., Backes, M., Wang, X. (eds.) ACM CCS 2018. pp. 896-912. ACM Press (Oct 2018)

[16] Boyle, E., Couteau, G., Gilboa, N., Ishai, Y., Kohl, L., Scholl, P.: Efficient pseudorandom correlation generators: Silent OT extension and more. In: Boldyreva, A., Micciancio, D. (eds.) CRYPTO 2019, Part III. LNCS, vol. 11694, pp. 489-518. Springer, Heidelberg (Aug 2019)

[17] Boyle, E., Gilboa, N., Ishai, Y.: Function secret sharing: Improvements and extensions. In: Weippl, E.R., Katzenbeisser, S., Kruegel, C., Myers, A.C., Halevi, S. (eds.) ACM CCS 2016. pp. 1292-1303. ACM Press (Oct 2016)

[18] Boyle, E., Goldwasser, S., Ivan, I.: Functional signatures and pseudorandom functions. In: Krawczyk, H. (ed.) PKC 2014. LNCS, vol. 8383, pp. 501-519. Springer, Heidelberg (Mar 2014) 
[19] Canetti, R.: Security and composition of multiparty cryptographic protocols. Journal of Cryptology 13(1), 143-202 (Jan 2000)

[20] Canetti, R.: Universally composable security: A new paradigm for cryptographic protocols. In: 42nd FOCS. pp. 136-145. IEEE Computer Society Press (Oct 2001)

[21] Chen, M., Cheng, C., Kuo, P., Li, W., Yang, B.: Multiplying boolean polynomials with frobenius partitions in additive fast fourier transform. CoRR abs/1803.11301 (2018)

[22] Damgård, I., Pastro, V., Smart, N.P., Zakarias, S.: Multiparty computation from somewhat homomorphic encryption. In: Safavi-Naini, R., Canetti, R. (eds.) CRYPTO 2012. LNCS, vol. 7417, pp. 643-662. Springer, Heidelberg (Aug 2012)

[23] Dessouky, G., Koushanfar, F., Sadeghi, A.R., Schneider, T., Zeitouni, S., Zohner, M. Pushing the communication barrier in secure computation using lookup tables. In: NDSS 2017. The Internet Society (Feb / Mar 2017)

[24] Doerner, J., shelat, a.: Scaling ORAM for secure computation. In: Thuraisingham, B.M., Evans, D., Malkin, T., Xu, D. (eds.) ACM CCS 2017. pp. 523-535. ACM Press (Oct / Nov 2017)

[25] Döttling, N., Ghosh, S., Nielsen, J.B., Nilges, T., Trifiletti, R.: TinyOLE: Efficient actively secure two-party computation from oblivious linear function evaluation In: Thuraisingham, B.M., Evans, D., Malkin, T., Xu, D. (eds.) ACM CCS 2017. pp. 2263-2276. ACM Press (Oct / Nov 2017)

[26] Druk, E., Ishai, Y.: Linear-time encodable codes meeting the gilbert-varshamov bound and their cryptographic applications. In: Naor, M. (ed.) ITCS 2014. pp. 169-182. ACM (Jan 2014)

[27] Esser, A., Kübler, R., May, A.: LPN decoded. In: Katz, J., Shacham, H. (eds.) CRYPTO 2017, Part II. LNCS, vol. 10402, pp. 486-514. Springer, Heidelberg (Aug 2017)

[28] Garg, S., Mahmoody, M., Masny, D., Meckler, I.: On the round complexity of OT extension. In: Shacham, H., Boldyreva, A. (eds.) CRYPTO 2018, Part III. LNCS, vol. 10993, pp. 545-574. Springer, Heidelberg (Aug 2018)

[29] Ghosh, S., Nielsen, J.B., Nilges, T.: Maliciously secure oblivious linear function evaluation with constant overhead. In: Takagi, T., Peyrin, T. (eds.) ASIACRYPT 2017, Part I. LNCS, vol. 10624, pp. 629-659. Springer, Heidelberg (Dec 2017)

[30] Gilboa, N., Ishai, Y.: Distributed point functions and their applications. In: Nguyen, P.Q., Oswald, E. (eds.) EUROCRYPT 2014. LNCS, vol. 8441, pp. 640-658. Springer Heidelberg (May 2014)

[31] Goldreich, O.: Foundations of Cryptography: Volume 2, Basic Applications. Cambridge University Press, New York, NY, USA (2004)

[32] Goldreich, O., Goldwasser, S., Micali, S.: How to construct random functions Journal of the ACM 33(4), 792-807 (Oct 1986)

[33] Goldreich, O., Micali, S., Wigderson, A.: How to play any mental game or A completeness theorem for protocols with honest majority. In: Aho, A. (ed.) 19th ACM STOC. pp. 218-229. ACM Press (May 1987)

[34] Hazay, C., Orsini, E., Scholl, P., Soria-Vazquez, E.: Concretely efficient large-scale MPC with active security (or, TinyKeys for TinyOT). In: Peyrin, T., Galbraith, S. (eds.) ASIACRYPT 2018, Part III. LNCS, vol. 11274, pp. 86-117. Springer, Heidelberg (Dec 2018)

[35] Hazay, C., Orsini, E., Scholl, P., Soria-Vazquez, E.: TinyKeys: A new approach to efficient multi-party computation. In: Shacham, H., Boldyreva, A. (eds.) CRYPTO 2018, Part III. LNCS, vol. 10993, pp. 3-33. Springer, Heidelberg (Aug 2018)

[36] Huang, Y., Evans, D., Katz, J.: Private set intersection: Are garbled circuits better than custom protocols? In: NDSS 2012. The Internet Society (Feb 2012)

[37] Ishai, Y., Kilian, J., Nissim, K., Petrank, E.: Extending oblivious transfers efficiently. In: Boneh, D. (ed.) CRYPTO 2003. LNCS, vol. 2729, pp. 145-161. Springer, Heidelberg (Aug 2003)

[38] Ishai, Y., Kushilevitz, E., Ostrovsky, R., Prabhakaran, M., Sahai, A.: Efficient noninteractive secure computation. In: Paterson, K.G. (ed.) EUROCRYPT 2011. LNCS, vol. 6632, pp. 406-425. Springer, Heidelberg (May 2011)

[39] Ishai, Y., Prabhakaran, M., Sahai, A.: Secure arithmetic computation with no honest majority. In: Reingold, O. (ed.) TCC 2009. LNCS, vol. 5444, pp. 294-314 Springer, Heidelberg (Mar 2009)

[40] Katz, J., Ranellucci, S., Rosulek, M., Wang, X.: Optimizing authenticated garbling for faster secure two-party computation. In: Advances in Cryptology - CRYPTO 2018 - 38th Annual International Cryptology Conference, Santa Barbara, CA USA, August 19-23, 2018, Proceedings, Part III. pp. 365-391 (2018), https://doi. org/10.1007/978-3-319-96878-0_13

[41] Keller, M., Orsini, E., Scholl, P.: Actively secure OT extension with optimal overhead. In: Gennaro, R., Robshaw, M.J.B. (eds.) CRYPTO 2015, Part I. LNCS, vol. 9215, pp. 724-741. Springer, Heidelberg (Aug 2015)

[42] Kiayias, A., Papadopoulos, S., Triandopoulos, N., Zacharias, T.: Delegatable pseudorandom functions and applications. In: Sadeghi, A.R., Gligor, V.D., Yung, M. (eds.) ACM CCS 2013. pp. 669-684. ACM Press (Nov 2013)

[43] Kilian, J.: Founding cryptography on oblivious transfer. In: Proceedings of the 20th Annual ACM Symposium on Theory of Computing, May 2-4, 1988, Chicago, Illinois, USA. pp. 20-31 (1988), https://doi.org/10.1145/62212.62215

[44] Kolesnikov, V., Kumaresan, R.: Improved OT extension for transferring short secrets. In: Canetti, R., Garay, J.A. (eds.) CRYPTO 2013, Part II. LNCS, vol. 8043, pp. 54-70. Springer, Heidelberg (Aug 2013)

[45] Lyubashevsky, V.: The parity problem in the presence of noise, decoding random linear codes, and the subset sum problem. In: Approximation, randomization and combinatorial optimization. Algorithms and techniques, pp. 378-389. Springer (2005)

[46] Mohassel, P., Rosulek, M.: Non-interactive secure 2PC in the offline/online and batch settings. In: Coron, J., Nielsen, J.B. (eds.) EUROCRYPT 2017, Part III. LNCS, vol. 10212, pp. 425-455. Springer, Heidelberg (Apr / May 2017)

[47] Naor, M., Pinkas, B.: Computationally secure oblivious transfer. Journal of Cryptology 18(1), 1-35 (Jan 2005)

[48] Naor, M., Pinkas, B.: Oblivious polynomial evaluation. SIAM J. Comput. 35(5), 1254-1281 (2006)

[49] Peikert, C., Vaikuntanathan, V., Waters, B.: A framework for efficient and composable oblivious transfer. In: Wagner, D. (ed.) CRYPTO 2008. LNCS, vol. 5157, pp. 554-571. Springer, Heidelberg (Aug 2008)

[50] Pinkas, B., Schneider, T., Segev, G., Zohner, M.: Phasing: Private set intersection using permutation-based hashing. In: Jung, J., Holz, T. (eds.) USENIX Security 2015. pp. 515-530. USENIX Association (Aug 2015)

[51] Pinkas, B., Schneider, T., Tkachenko, O., Yanai, A.: Efficient circuit-based psi with linear communication (2019), https://eprint.iacr.org/2019/241

[52] Prange, E.: The use of information sets in decoding cyclic codes. IRE Transactions on Information Theory 8(5), 5-9 (1962)

[53] Sendrier, N.: Decoding one out of many. In: Yang, B.Y. (ed.) Post-Quantum Cryptography - 4th International Workshop, PQCrypto 2011. pp. 51-67. Springer, Heidelberg (Nov / Dec 2011)

[54] Torres, R.C., Sendrier, N.: Analysis of information set decoding for a sub-linear error weight. In: Takagi, T. (ed.) Post-Quantum Cryptography - 7th International Workshop, PQCrypto 2016. pp. 144-161. Springer, Heidelberg (2016)

[55] Wang, X., Ranellucci, S., Katz, J.: Authenticated garbling and efficient maliciously secure two-party computation. In: Thuraisingham, B.M., Evans, D., Malkin, T., Xu, D. (eds.) ACM CCS 2017. pp. 21-37. ACM Press (Oct / Nov 2017)

[56] Wang, X., Ranellucci, S., Katz, J.: Global-scale secure multiparty computation. In: Thuraisingham, B.M., Evans, D., Malkin, T., Xu, D. (eds.) ACM CCS 2017. pp. 39-56. ACM Press (Oct / Nov 2017)

[57] Zichron, L.: Locally computable arithmetic pseudorandom generators. Master's thesis, School of Electrical Engineering, Tel Aviv University (2017), http://www. eng.tau.ac.il/ bennyap/pubs/Zichron.pdf 